\title{
Estudio geoarqueológico de la cueva de El Sidrón (Piloña, Asturias)
}

\author{
J.C. Cañaveras ${ }^{(1)}$, S. Sánchez-Moral ${ }^{(2)}$, S. Cuezva ${ }^{(3)}$, A. Fernández-Cortés ${ }^{(3)}$, M.C. Muñoz ${ }^{(1)}, J$. Lario ${ }^{(4)}$, \\ P.G. Silva ${ }^{(5)}$, G. Santos-Delgado ${ }^{(6)}$, E. Duarte ${ }^{(7)}$, D. Santamaría ${ }^{(7)}$ y M. de la Rasilla ${ }^{(7)}$
}

(1) Departamento de Ciencias de laTierra y del Medio Ambiente, Universidad de Alicante, Campus San Vicente del Raspeig, E-03080 Alicante, España Jc.canaveras@ua.es; mc.munoz@ua.es

(2) Departamento Geología, Museo Nacional de Ciencias Naturales, CSIC, José Gutiérrez Abascal 2, E-28040 Madrid, España ssmilk@mncn.csic.es; scuezva@mncn.csic.es

(3) Departamento de Biología y Geología. Universidad de Almería, España scuezva@ual.es; acortes@ual.es

(4) Departamento de Ciencias Analíticas, UNED, Paseo de la Senda del Rey 9, E-28040 Madrid, España javier.lario@ccia.une.es

(5) Departamento de Geología, Universidad de Salamanca, Plaza de los Caídos s/n, E-37008 Salamanca, España pgsilva@usal.es

(6) Departamento de Ingeniería Cartográfica y del Terreno, Facultad de Ciencia, Universidad de Salamanca, Plaza de la Merced s/n, E-37008 Salamanca, España gsd@usal.es

(7) Departamento de Historia. Universidad de Oviedo, Teniente Alfonso Martínez s/n, E-33011 Oviedo santamariadavid@uniovi.es; elduarma@gmail.com; mrasilla@uniovi.es

\begin{abstract}
RESUMEN
El relleno sedimentario que alberga el registro arqueológico y antropológico de la Galería del Osario (cueva de El Sidrón) presenta una alta complejidad, tanto en los tipos de sedimentos como en su distribución espacial en la cavidad. Los principales factores que rigen esta complejidad son la naturaleza episódica y altamente energética de gran parte de las unidades litoestratigráficas reconocidas y la irregular geometría del propio conducto, que compartimenta significativamente las áreas de deposición. A modo de resumen puede indicarse que en la Galería del Osario quedan representados todos los tipos de materiales detríticos típicos de depósitos alóctonos en ambiente kárstico; desde materiales bien seleccionados de granulometría fina representativos de facies de encharcamiento (arcillas de la unidad O) o de desbordamiento (Unidad IV), a depósitos groseros mal seleccionados correspondientes a coladas de barro y detritos en masa (Unidad II y base de unidad III), pasando por típicas facies fluvio-kársticas o de canal compuestas por materiales arenosos con laminaciones y estructuras de flujo (unidades I y II). Los restos óseos se concentran en la unidad III. El área fuente se sitúa en cotas superiores a la Galería del Osario, es decir, en los niveles intermedio o superior del sistema kárstico, y el depósito o entrada de material óseo a la Galería del Osario se activaría en episodios de alta energía relacionado con eventos de inundación y/o tormenta.
\end{abstract}

Palabras clave: Cueva de El Sidrón, Geoarqueología, karst, neandertal, Musteriense.

\section{Geoarchaeological study of the Sidrón cave (Piloña, Asturias)}

\begin{abstract}
The sedimentary infill bearing the archaeological and anthropological record of the Ossuary Gallery (the Sidrón cave) shows high complexity in both sediment types and their spatial distribution in the cavity. The main factors that govern this complexity are the episodic and highly energetic nature of the recorded sedimentary infill units and the irregular geometry of the gallery itself, which significantly compartmentalized areas of deposition. Briefly, all types of typical allochthonous detrital material deposits in karst environments are represented in the Ossuary Gallery; from well-sorted fine-grained sediment facies representative of backswamp (clays of the unit O) or slackwater (Unit IV), to poorly-sorted coarse-grained deposits corresponding to mudflows and debris flows (Unit II and Unit III base), and typical channel facies composed of laminated sands
\end{abstract}


Cañaveras, J.C., et al., 2018. Estudio geoarqueológico de la cueva de El Sidrón... Boletín Geológico y Minero, 129 (1/2): 107-128

and silts (units I and II). The bone and lithic remains are concentrated in unit III. The source area is above the Ossuary Gallery, at the intermediate or higher karst levels. The input of archeological material to the Ossuary Gallery was triggered in high energy episodes in association to flood events and / or storms.

Key words: El Sidrón Cave, Geoarchaeology, karst, Neanderthal, Mousterian.

\section{ABRIDGED ENGLISH VERSION}

\section{Introduction and methods}

The excavation and study of Neanderthal fossils and Mousterian lithics from the Sidrón Cave (Asturias, northern Spain) is an example of interdisciplinary geoarchaoelogical research (Rasilla et al, 2011; 2014). Regulated archaeological excavations in the Sidrón Cave began in 2000. Since then, a group of specialists in various earth science disciplines (geologists, anthropologists, archaeologists,...) have carried out the study and interpretation of the archaeological and anthropological record that has appeared in the cave. The bone assemblage in the Sidrón Cave is almost exclusively Neanderthal human remains ( 2530): A total of 13 individuals have so far been identified. There are almost no animal bones. A small assemblage of lithic artifacts ( 400), representing a Mousterian assemblage, have also been recovered, all made from local rocks, mostly chert and quartzite. Preservation of the bones is excellent, with very limited trampling or erosion and no large carnivore toothmarks. The archaeological assemblage is not in its original location, the human remains and lithics were dropped into the cave in a single event via a collapse of nearby conduits above the site. The age of the Neanderthal remains found in the Sidrón cave has been accurately fixed at 48,400 3200 BP (Rasilla et al., 2013 and references herein).

The Sidrón cave is developed in Oligocene carbonate conglomerates alternating with fine- to mediumgrained sandstones (Fig. 1). The carbonate succession is in an approximately E-W direction, dipping 20-30 to the north. The karst system, with a development of $600 \mathrm{~m}$ (about $3700 \mathrm{~m}$ in galleries) and a height of 30$32 \mathrm{~m}$ among the highest galleries and the spring, is divided into four levels with a main E-W guideline, which were generated according to the evolution of the regional drainage system. In the second level, just above the active (phreatic) level, the Main Gallery (Gallery of the River) and its transverse tributaries (i.e. Ossuary Gallery) are located. The Neanderthal collection is located in the Ossuary Gallery, a N-S oriented passage, 28 $\mathrm{m}$ long and $12 \mathrm{~m}$ wide. Each endokarst level is reflected on the outside (exokarst) as terraces and other surface landforms that form the Depression of the Sidrón, an E-W blind valley or polje whose main stream enters the current karst system in its eastern side (Fig.16). This depression is developed along the geological contact between the Cretaceous calcareous materials and Paleogene conglomerate overlying formation in which the endokarstic system is developed.

The geological and geomorphological analysis of the area allowed the framing of the karst system in the geological context of the study area. Georeferenced endo- and exokarst topography has served as the basis for a detailed geomorphological analysis. Since 2000 we have proceeded to record, sample, analyse and study all the lithostratigraphic profiles and sections discovered during archaeological excavations that were carried out. The geological study of the interior of the cave was not limited to the analysis of sedimentary infill. In order to explore the roof of the Ossuary Gallery and verify the existence of possible source areas of sediments from higher galleries several speleological campaigns were conducted. The characterization of materials begins with in situ description of the sediments and a systematic sampling. Sediment grain size distribution were achieved by sieving, sedimentation balance and laser coulter analyzer. Petrographic observations are based on the examination of polished thin sections by transmitted light microscopy. The mineralogy of samples was determined using a Philips XRD system operating at $40 \mathrm{kV}$ and $30 \mathrm{~mA}$ with monochrome CuKa radiation.

\section{Results and discussion}

The sedimentary infill in the Ossuary Gallery shows great complexity and thus makes it hard to define a stratigraphic column representing the whole gallery. In the central zone of Ossuary Gallery, five main units corresponding to events with different hydrodynamic and sedimentary characteristics are defined (Sánchez-Moral et al. 2007; Cañaveras et al., 2011) (Fig. 15). From bottom to top (Figs. 4 and 7):

0 : Unit of massive mud located at the bottom of the column. No clear sedimentary structures can be distinguished. Provisionally they appear to be sediments deposited through a low energy outflow or backswamp conditions.

l: Unit of laminated fine sands and mud, with cross-stratification. It includes low-intensity fluvial-karstic material with a relative increase in energy at the top. 
Cañaveras, J.C., et al., 2018. Estudio geoarqueológico de la cueva de El Sidrón... Boletín Geológico y Minero, 129 (1/2): 107-128

II: Unit of poorly sorted gravels, sands and mud. It has so far represented the lower limit of the fossiliferous units. The fluvial-karstic materials originated from a high energy event and are clearly erosive in nature. This unit corresponds to diamicton facies.

III: Unit of massive clays with dispersed levels of gravels, sands and silts. Interbedded silts and fine sands showing waterscape structures are common. At the base, this unit is very similar to the previous one and in general the grain size diminishes towards the top. In the western part of the gallery, the grain size of the unit is also coarser, with a predominance of pebble and gravel deposits. A prominent feature is the existence of calcareous crusts (IIIC) on the top of the unit.

IV: Unit of massive mud with some interbedded sands. These sediments formed in a very low energy fluvialkarstic environment, and correspond to the final infill episode in the gallery which can be regarded as still in progress.

The vast majority of the anthropological and archaeological material is concentrated between frames $E$ $H / 10-E-H / 4$ in Unit III (Figs. 3 and 8). There are a few remains, with a slight increase in the number of fauna, which is always scarce, in the southern part of the gallery (D-E-F/20-23). The geological analysis of the sediments suggests that the bones dropped into the cave via a vertical shaft, in a massive flow deposit, probably resulting from a flood event after a thunderstorm (Fig. 12). Several pieces of evidence suggest that the archaeological and anthropological remains were deposited near-simultaneously shortly before the high energy event: marks left by the gnawing of large carnivores are absent, articulated Neanderthal bones are present, 20\% of the associated lithic industry can be refitted (Santamaría et al. 2010; 2012). Moreover, the good condition of the bones indicates that these come from outside, but must have been in a protected environment (e.g., a surficial gallery near the exterior or a rock shelter) and the time exposure in surface conditions was short, given the scant traces of alteration of the pieces.

At the bottom or southern zone of the Ossuary Gallery autochthonous (collapses) and allochthonous (colluvial, debris flow, fluvio-karst) deposits are recognized (Figs. 10 and 11). The scarce remains of macrofauna entered via another channel that took them to the southern part of the gallery.

The Cimera Gallery is part of the upper level of the four in which the Sidrón karst system is structured (Fig. 13). The sedimentary record in this gallery show the existence of high-energy events (massive gravels) sealed by slackwater facies (laminated clays) (Fig. 14).

The Ossuary Gallery morphology (width, sinuosity,...) has influenced the hydrodynamic behaviour of the cavity, resulting in steep energy gradients, which are reflected in the complex distribution of different sediment facies. The special configuration of the bottom of the gallery (sponge-work), has been responsible for the complex geometry of its sediment infill, but, in turn, has favoured the preservation of archaeological material. Many of these fossiliferous deposits have been well protected from episodes of sediment reworking and destruction, so common in karst dynamics.

\section{Introducción}

La Geoarqueología ha dejado de ser un mero apoyo a la investigación arqueológica a desarrollarse como una disciplina científica multidisciplinar clave para interpretar las relaciones entre hombre y medioambiente plasmadas en el registro arqueológico (Jordá Pardo, 2009). La aplicación, de manera sistemática e integrada, de métodos y técnicas procedentes de disciplinas de las Ciencias de la Tierra resulta indispensable para la contextualización de yacimientos prehistóricos en contextos de cueva y abrigo con el fin de definir su evolución paleoambiental (Farrand 2001; Woodward et al., 2001; Goldberg y Macphail, 2005; Goldberg y Sherwood, 2006).

La excavación y estudio del yacimiento con fósiles neandertales e instrumentos líticos musterienses de la cueva de El Sidrón es un ejemplo de investigación geoarqueológica interdisciplinar (Rasilla et al., 2011; 2014). Las excavaciones arqueológicas regladas en la cueva de El Sidrón comenzaron en el año 2000, des- pués de un complejo proceso judicial y administrativo que se inició en 1994 con el hallazgo fortuito de los primeros restos óseos en la cueva (Fortea et al., 2003; 2007a). Desde entonces un grupo de especialistas en diferentes disciplinas y técnicas analíticas (geólogos, antropólogos, arqueólogos, genetistas...) han llevado a cabo el estudio y la interpretación del registro arqueológico y antropológico que ha aparecido en EI Sidrón. A lo largo de estos años las intervenciones desarrolladas en El Sidrón se han ido solapando según los resultados progresivamente obtenidos y las necesidades de investigación a partir de los datos y de los interrogantes planteados (Rasilla et al., 2011)

El conjunto arqueológico de la cueva de El Sidrón está formado fundamentalmente por restos humanos ( 2530), pertenecientes a la especie Homo neanderthalensis (Rosas y Aguirre 1999; Prieto et al.,2001; Fortea et al., 2003; 2007b; Rosas et al., 2006; 2013), y líticos ( 400 piezas) (Santamaría, 2012), más unos pocos restos de macrofauna ( 100). Entre el registro óseo destacan cuatro mandíbulas, tres maxilares, 
multitud de dientes, fragmentos craneales y diferentes huesos del tronco y de las extremidades, correspondientes, al menos, a 13 individuos (siete adultos, tres adolescentes, dos juveniles y un infantil) (Fortea et al., 2003; Rosas et al., 2006; 2007; 2013; Rasilla et al., 2011; 2014). Del número total de restos líticos, que representan una colección musteriense, 61 de ellos son útiles, 185 productos de lascado brutos, 9 bases de lascado, 1 núcleo-útil y el resto dudosos (Santamaría, 2012, Santamaría et al., 2011).

La edad de los restos neandertales y sedimentos asociados en la Galería del Osario ha sido obtenidas por diversos métodos de datación (Torres et al., 2010; Rasilla et al., 2013; Wood et al., 2013): ${ }^{14} \mathrm{C}$ AMS se ha aplicado a restos óseos, OSL a sedimentos detríticos finos (ricos en cuarzo), ESR a sedimentos y AAR a conchas y dentina. Dataciones por U/Th aplicadas a materiales carbonáticos (espeleotemas) no han dado resultados, dada la alta contaminación de torio residual en todas las muestras. La ponderación de los resultados obtenidos por los diversos procedimientos empleados ha dado unas edades para los restos antropológicos y los sedimentos que los contienen (principalmente unidad III) en torno a 49.000 años de antigüedad. En cuanto a la edad del resto de las unidades litoestratigráficas, las unidades I y IV han sido datadas por OSL, dando unas edades de unos 47000 y $28000 \pm 2500$ a BP, respectivamente. El techo de la unidad III ha dado una antigüedad de aproximadamente 30000 años por el mismo método.

En el presente artículo se muestran algunos de los resultados de los trabajos geológicos (estratigrafía, sedimentología, mineralogía, geomorfología...) realizados en la cueva de El Sidrón, como investigación y apoyo a los trabajos de prospección y excavación arqueológica.

\section{Zona de estudio}

El complejo kárstico de la cueva de El Sidrón se desarrolló en el seno de un macizo rocoso de edad paleógena formado por una sucesión de conglomerados calcáreos muy cementados entre los que se intercalan niveles de areniscas y arcillas arenosas menos resistentes (Fm. Pudinga de Posada). Todos estos materiales presentan un buzamiento generalizado de unos $20-30^{\circ} \mathrm{N}$ y están afectados por diversas fallas y fracturas con direcciones predominantes $\mathrm{E}-\mathrm{O}$ y N-S.

El sistema kárstico, con un desarrollo de unos 600 $\mathrm{m}$ y un desnivel de 30-32 $\mathrm{m}$ entre las galerías más altas (Galería Cimera, $172 \mathrm{~m}$ ) y la surgencia (Salelagua, $142 \mathrm{~m}$ ), se estructura en cuatro niveles (unos $3700 \mathrm{~m}$ en galerías) con una dirección principal
$\mathrm{E}-\mathrm{O}$, que se generaron según progresaba el encajamiento de la red fluvial regional que gobierna los procesos de espeleogénesis y controla su evolución temporal. En el segundo de los niveles, justo por encima del nivel activo, predominantemente freático, se sitúan la Galería Principal o Galería del Río y sus tributarios transversales, como la Galería del Osario que es donde se encuentra el registro arqueológico y antropológico de la cueva (Fig. 1).

La naturaleza más o menos soluble o resistente a la erosión de los cuerpos de roca que forman el encajante del sistema de El Sidrón, junto con el grado de deformación de las mismas (plegamiento y fracturación) determinan la compleja morfología de conductos y cavidades que caracteriza el laberinto subterráneo jerarquizado que constituye el sistema kárstico de El Sidrón.

La evidente relación de las cavidades con la tectónica queda bien reflejada en la propia morfología de la Galería del Osario, que se dispone transversalmente $\left(\mathrm{N} 20^{\circ} \mathrm{E}\right)$ a la Galería Principal (E-O) que va paralela a la dirección de las capas según el trazado de la falla regional más importante. La Galería del Osario se alinea con fallas trasversales perpendiculares a los estratos a favor de la inclinación hacia el $\mathrm{N}$ de los mismos.

Cada uno de estos niveles endokársticos se ve reflejado en el exterior (exokarst) en forma de terrazas y otras formas superficiales del terreno que configuran la Depresión de El Sidrón, un poljé o valle ciego de dirección E-O cuyo arroyo principal se sume por su extremo oriental en el interior del sistema kárstico actual (Silva et al., 2011, Santos-Delgado et al., 2012b). Esta depresión se desarrolla a lo largo del contacto geológico existente entre los materiales calcáreos cretácicos y la formación de conglomerados paleógenos suprayacentes en los que se ha desarrollado el sistema de galerías subterráneas o endokarst antes mencionado.

Son abundantes cantos calcáreos con pátina negra de Fe y $\mathrm{Mn}$ registro sedimentario de la cavidad (derivados de la Pudinga de Posada). Su depósito dentro de la cueva en grandes cantidades durante el Paleolítico Inferior (Fortea et al., 2003) probablemente se deba a la erosión en el Pleistoceno de los conglomerados cenozoicos (Pudinga de Posada) (Martínez García, 2011).

\section{Metodología}

El análisis geológico y geomorfológico de la zona ha permitido situar el complejo kárstico del Sidrón en el contexto geológico del área de estudio. Los trabajos 

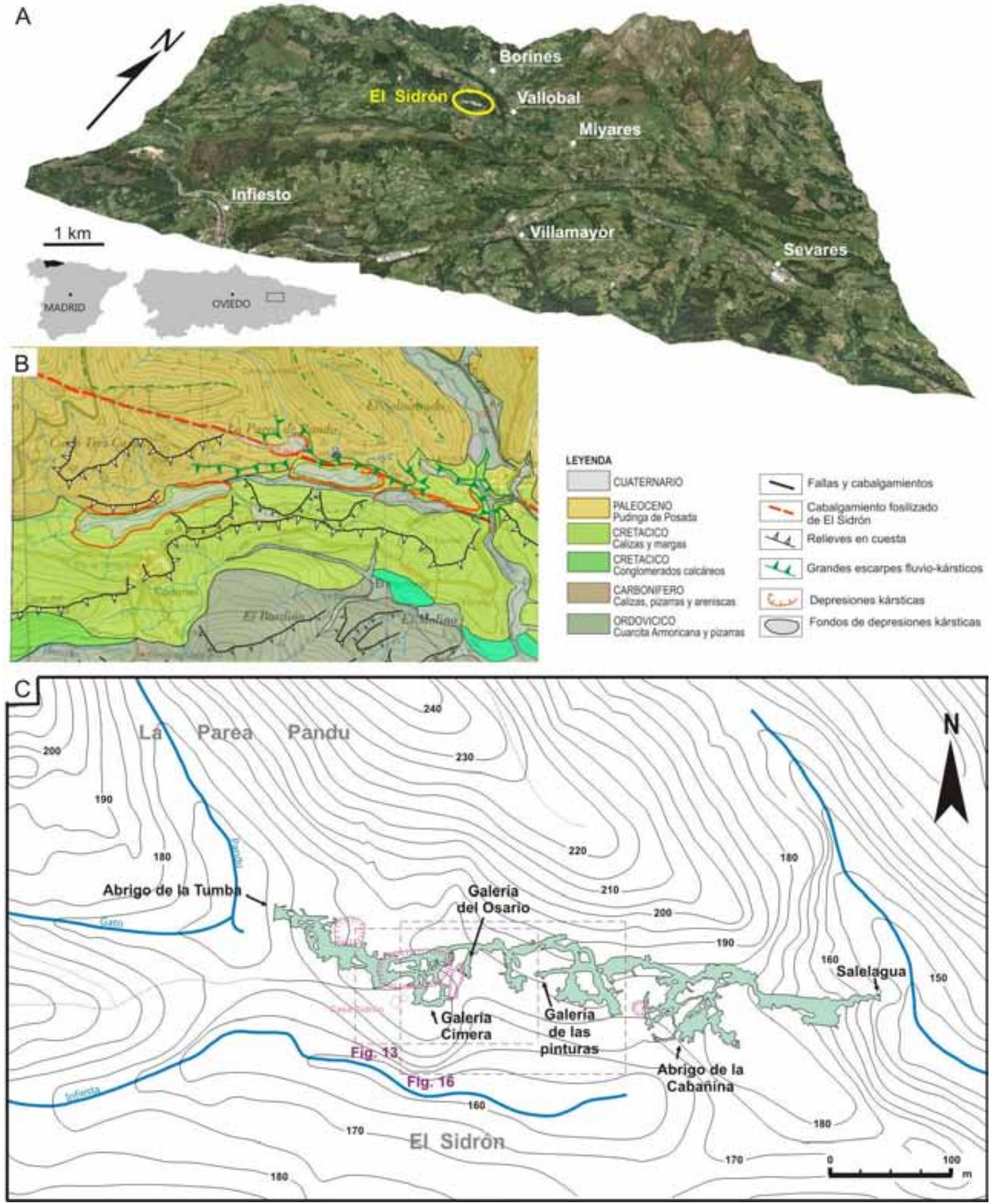

Figura 1. A) Localización geográfica de la cueva de El Sidrón. B) Esquema geológico del area de El Sidrón. C) Plano de la cueva con la situación de las principales galerías. Se indica a que parte de la cueva corresponden los planos de las figuras 13 y 16.

Figure 1. A) Geographical location of the Sidrón cave. B) Geological map of the Sidrón area. C) Cave map with the location of the main galleries. Extension of maps from both Figures 13 and 16 are indicated. 
topográficos han abarcado la totalidad de la depresión kárstica de El Sidrón confeccionando un mapa a escala 1:1.000 con curvas de nivel cada metro en el que se ha georreferenciado la topografía de la cavidad kárstica interior y que ha servido de base para el análisis geomorfológico de detalle. Para georreferenciar la cavidad interior y localizarla respecto a las zonas de prospección exterior se han utilizado métodos de topografía clásica, enlazando poligonales exteriores e interiores convenientemente cerradas (Silva et al., 2011; Santos-Delgado et al., 2012a y Santos-Delgado et al., 2012b).

Desde 2000 se ha procedido a la testificación, muestreo, análisis y estudio de todos los perfiles arqueológicos descubiertos durante las campañas de excavación Ilevadas a cabo en la cueva de El Sidrón y su entorno (Fortea et al., 2003; Sánchez-Moral et al., 2007; Cañaveras et al., 2011).

La caracterización de los materiales se inicia con una descripción in situ de los mismos que constituyen las secciones y continua con un muestreo sistemático de las mismas. Con posterioridad en el laboratorio se ha realizado una caracterización detallada de los materiales consistente en:

- Análisis granulométricos por medio del cribado y tamización en seco de la muestra total y el empleo de balanza de sedimentación para la fracción fina o lutítica (limos y arcillas). En muestras seleccionadas, y con el objeto de analizar en detalle la fracción fina, se realizó un análisis de alta resolución de la fracción menor de $2 \mathrm{~mm}$ mediante un contador láser, basado en la difracción de la luz emitida por un láser.

- Análisis mineralógicos mediante Difracción de Rayos X (Espectrómetro DRX Philips PW 1710; laboratorio MNCN-CSIC) en muestra de polvo para sacar la composición mineralógica global. En muestras finas (limos y arcillas) se han realizado difractogramas de polvo total y un análisis de agregados orientados (A.O.).

- Análisis petrográfico de la roca encajante y de las costras carbonáticas asociadas al relleno detrítico mediante el estudio de láminas delgadas por técnicas de microscopía óptica de polarización. Para las descripciones petrológicas de rocas carbonáticas se ha utilizado la clasificaciones de Dunham (1962) y Folk (1962), mientras que para rocas detríticas se ha utilizado la clasificación de Pettijohn et al. (1973).

En algunas campañas además de la caracterización de los materiales por las técnicas expuestas, se ha realizado un análisis geoquímico (elementos mayores y trazas, siendo los más representativos $\mathrm{Si}$, $\mathrm{Ti}, \mathrm{Ca}, \mathrm{Fe}, \mathrm{Mn}, \mathrm{S}, \mathrm{Zn}, \mathrm{Zr}$, Sr y Y) de muestras seleccio- nadas con técnicas de Fluorescencia de Rayos $\mathrm{X}$ (FRX, espectrómetro Phillips modelo PW-2424 con un tubo de rayos $X$ de ventana ultra-fina $y$ ánodo de rodio ( $\mathrm{Rh})$ a 2,4 kW; laboratorio $\mathrm{MNCN}-\mathrm{CSIC}$ ). Asimismo se han realizado análisis de Magnetismo Ambiental mediante la realización de medidas de la susceptibilidad magnética de los sedimentos acumulados in situ (equipo Bartington modelo MS2) y en laboratorio (laboratorios IPNA-CSIC).

El estudio geológico del interior de la galería no se limitó al análisis del relleno. Con el objeto de explorar el techo de de la galería y comprobar la existencia o ausencia de posibles zonas de aporte de material procedentes de galerías colgadas y situadas por encima de la del Osario se realizaron varias campañas espeleológicas.

\section{Resultados y discusión}

\section{Roca encajante}

Como se ha apuntado anteriormente, el sistema kárstico de la cueva de El Sidrón se desarrolla en el seno de una secuencia de conglomerados y areniscas oligocenas (Fm. Pudinga de Posada, Oligoceno). Los conglomerados están constituidos por cantos calizos (biopelmicritas y biopelsparitas) procedentes del Santoniense de tamaño variable (centimétricos a decimétricos). La proporción de cemento es variable y generalmente de tipo mosaico equant o microcristalino. Las areniscas están constituidas por arenitas (litarenitas, sublitarenitas y subarcosas) y grauwackas caracterizadas por una trama inequigranular, pobremente seleccionado, compuesto por granos angulosos a subangulosos de cuarzo mono- y policristalino y fragmentos de roca como elementos mayoritarios. Entre estos últimos destaca la abundancia de fragmentos de roca metamórfica (cuarcitas principalmente) y de roca sedimentaria como agregados tipo grainstone y/o packstone fosilíferos, así como fragmentos de bioclastos (bivalvos, foraminíferos, equinodermos,...), más o menos grandes y reelaborados, como agregados tipo grainstone y/o packstone fosilíferos.

Durante la campaña arqueológica de 2006 se realizaron una serie de sondeos de prospección geológica en el exterior (epikarst), justo en la vertical de la Galería del Osario, con el objeto buscar zonas de rellenos intra-kársticos que correspondieran con posibles zonas de aporte directo de material a la zona prospectada en el interior de la galería en campañas previas (Fig. 2).

En conjunto, los resultados indican potentes relle- 

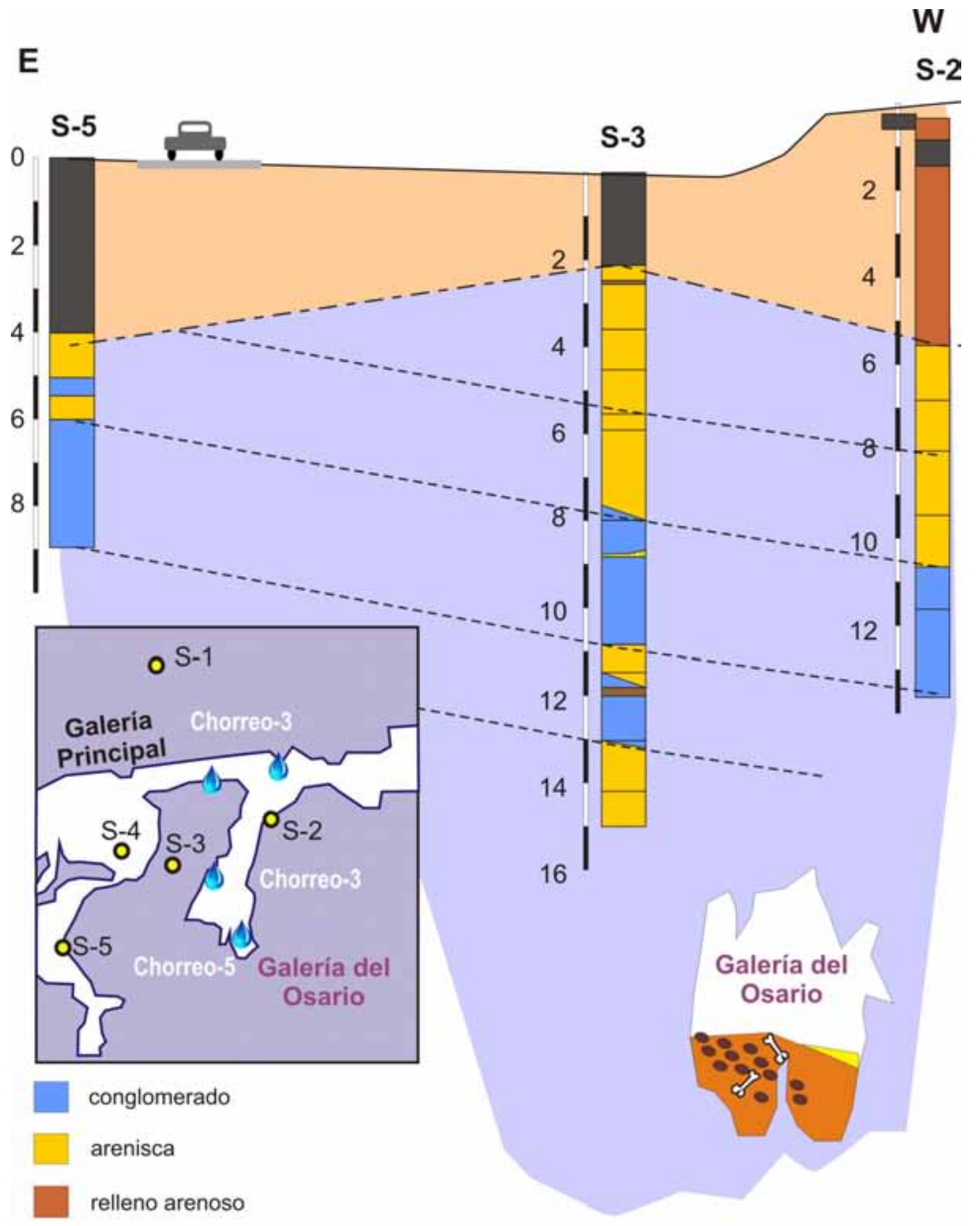

Figura 2. Correlación de los sondeos S-2, S-3 y S-5 en un perfil E-O, indicando la posición relativa de la zona de la Galería del Osario. Se indica dónde percolaron ("chorreos") los lodos de sondeo en el interior de la cueva durante las labores en 2006.

Figure 2. Correlation of S-2, S-3 and S-5 mechanical boreholes in an E-W profile, indicating the position of Ossuary Gallery. Localization of percolated drilling water ("drips") inside the cave during survey work in 2006 is indicated. 
nos arcillosos y bancos de areniscas alterados (descementados) en la zona superior y la alternancia de paquetes de areniscas y conglomerados con espesor variable (1 a $5 \mathrm{~m}$ ) constituyendo la roca encajante de la cavidad. En el sondeo S-3 a los $8,8 \mathrm{~m}$ se identificó un espeleotema macroesparítico y una superficie de discontinuidad activa con circulación de agua que indican la actividad kárstica durante un intervalo de tiempo (formación del espeleotema) y hasta la actualidad (circulación de agua) en niveles situados inmediatamente en la vertical de la Galería del Osario. Los puntos de infiltración de agua (denominados "chorreos" en Fig. 2), derivadas de las labores de sondeo, localizados en la Galería Principal y en la Galería del Osario, señalan asimismo que existe conexión real entre los niveles de roca perforados y el interior de la cavidad.

\section{Morfología de la Galería del Osario}

La Galería del Osario, donde se han centrado la mayor parte de los trabajos geoarqueológicos es una pequeña galería que se dispone transversalmente al sistema principal. Se trata de una galería estrecha con orientación prácticamente $\mathrm{N}^{\circ} 0^{\circ} \mathrm{E}$, de planta tabular, ramificada y estrechada en su parte sur y que alcanza una altura considerable, superando los 5 metros en su zona central (Alonso Peña, 2011; Santos-Delgado et al., 2012a; Santos-Delgado et al., 2012b) (Fig. 3A). En el techo se reconocen morfologías de disolución, principalmente pequeñas cúpulas elipsoidales y tubos más o menos inclinados; así como entradas obturadas por sedimentos que comunican con galerías (simas o niveles) superiores. Estas estructuras se disponen de forma alineada en el techo con unas direcciones paralelas a las de la propia galería delineando trazas de fracturas.

La inclinación del conducto y las trazas de fracturas a techo denotan claramente el control estratigráfico y estructural de su formación. Una característica prominente de esta galería es la existencia en techo y suelo (tal y como se ha ido manifestando con el avance de las excavaciones) de espeleogenes o morfologías espadiformes labradas en el conglomerado encajante con una orientación predominante N-S. Estas morfologías en el suelo de la cavidad en forma de tabiques o paredes, configuran un abrupto lapiaz interno o spongework grosero, compuesto por tabiques o espadones de forma irregular que influyen en gran medida en la hidrodinámica del conducto y actúan como trampas o compartimentos para la sedimentación controlando la arquitectura deposicional del mismo. La existencia de estas meso y micromorfolo- gías características formadas por disolución y/o erosión es indicativa de que la espeleogénesis de esta cavidad se produjo mediante procesos de ensanchamiento por disolución a favor de una zona fracturada y no de una fractura o falla simple (Cañaveras et al., 2011).

Se exploraron también todos los posibles conductos de conexión de la Galería del Osario con un hipotético nivel superior del que podrían proceder los restos óseos y la industria lítica. En muchos de los casos se corroboró que la mayor parte de esos conductos sin salida y producidos en condiciones freáticas no muestran continuidad con niveles superiores y acaban en roca caja. No obstante algunos de los conductos presentan sedimentos detríticos y espeleotémicos.

\section{Sedimentología}

Las excavaciones en el interior de la cueva de EI Sidrón se han centrado en la Galería del Osario, que es donde se han recuperado la totalidad de los fósiles neandertales e instrumentos líticos recogidos hasta el momento, y en varias galerías y conductos limítrofes (p. ej. Galería de los Caracoles, Zona Salomón-Galería Cimera).

\section{Galería del Osario}

Los primeros restos óseos extraídos de la galería en 1994, y posteriormente todos los demás desde el inicio de las excavaciones regladas en 2000 se localizan en la parte centro-septentrional de la galería (cuadrículas E-F-G-H/5-10; cortes 2, 3 y 5) (Fig. 3B).

El avance de las excavaciones arqueológicas en esta zona ha permitido definir una columna tipo del relleno de la Galería a partir de niveles sedimentarios con características similares entre sí, a lo largo de todos los perfiles abiertos. Hasta el momento el relleno se ha dividido en cinco niveles principales (Figs. 4 y 5).

Unidad O (basal): Formada por materiales granulares finos (limos y arcillas masivas) de coloración rojiza, de un espesor mínimo de $30 \mathrm{~cm}$ y sin estructuras sedimentarias claras. El tamaño de grano disminuye hacia el sur, alejándose de la Galería Principal, y presumiblemente también lo haga hacia techo, indicando un episodio de baja energía y/o energía decreciente a techo. No contiene restos arqueológicos 0 antropológicos.

Unidad I: Constituida por arenas finas y limos interlaminados, con estratificación cruzada (de surco 
A
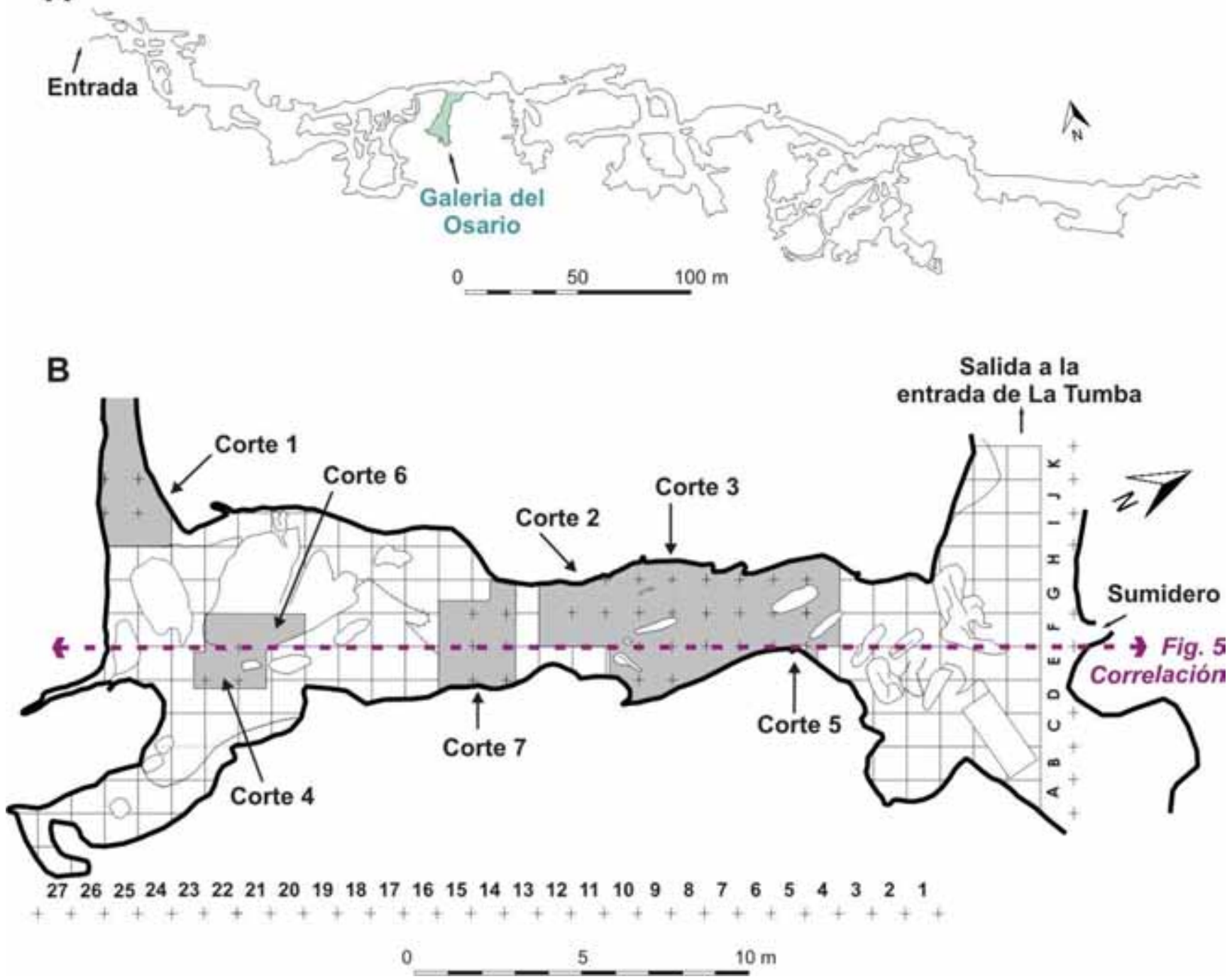

Figura 3. A) Plano de la cueva de El Sidrón y B) de la Galería del Osario, con indicación de la cuadrícula de excavación y la cronología de los cortes litoestratigráficos estudiados.

Figure 3. Map of the Sidrón cave $(A)$ and the Ossuary Gallery $(B)$, indicating the excavation grid and the location of the lithostratigraphic sections studied.

hacia la base de la unidad). Las láminas presentan un espesor de orden milimétrico a centimétrico y una estructura interna grano decreciente (arenas finas a muy finas o limos). Tiene una coloración amarillenta, con tonos más claros cuanto mayor es el tamaño de grano de las láminas. De potencia variable en las diversas secciones estudiadas $(30-70 \mathrm{~cm})$, no se ha reconocido en las zonas más septentrionales de la galería (hacia la Galería Principal). No contiene restos arqueológicos ni antropológicos.

Unidad II: Constituida por gravas finas y arenas gruesas a muy gruesas. Se trata de materiales mal seleccionados, generalmente grano-soportados que presenta un carácter netamente erosivo sobre la uni- dad subyacente. Localmente es muy notable y característica la abundancia de cantos con pátinas negras. Esta unidad supone, hasta el momento, el límite inferior de las unidades fosilíferas. La geometría del depósito, con marcada pendiente y aumento de espesor hacia el $\mathrm{N}$ y $\mathrm{O}$ marca claramente la dirección de los aportes. En la figura 6 se muestra una síntesis de los principales parámetros granulométricos, mineralógicos y magnéticos de uno de los cortes estudiados en la zona F-G / 8. En general, se puede ver cómo la Unidad II muestra un brusco incremento de la susceptibilidad magnética, asociada a la presencia de gravas con costra ferruginosa milimétrica y marcando un evento de ruptura en el relleno de la Galería del 


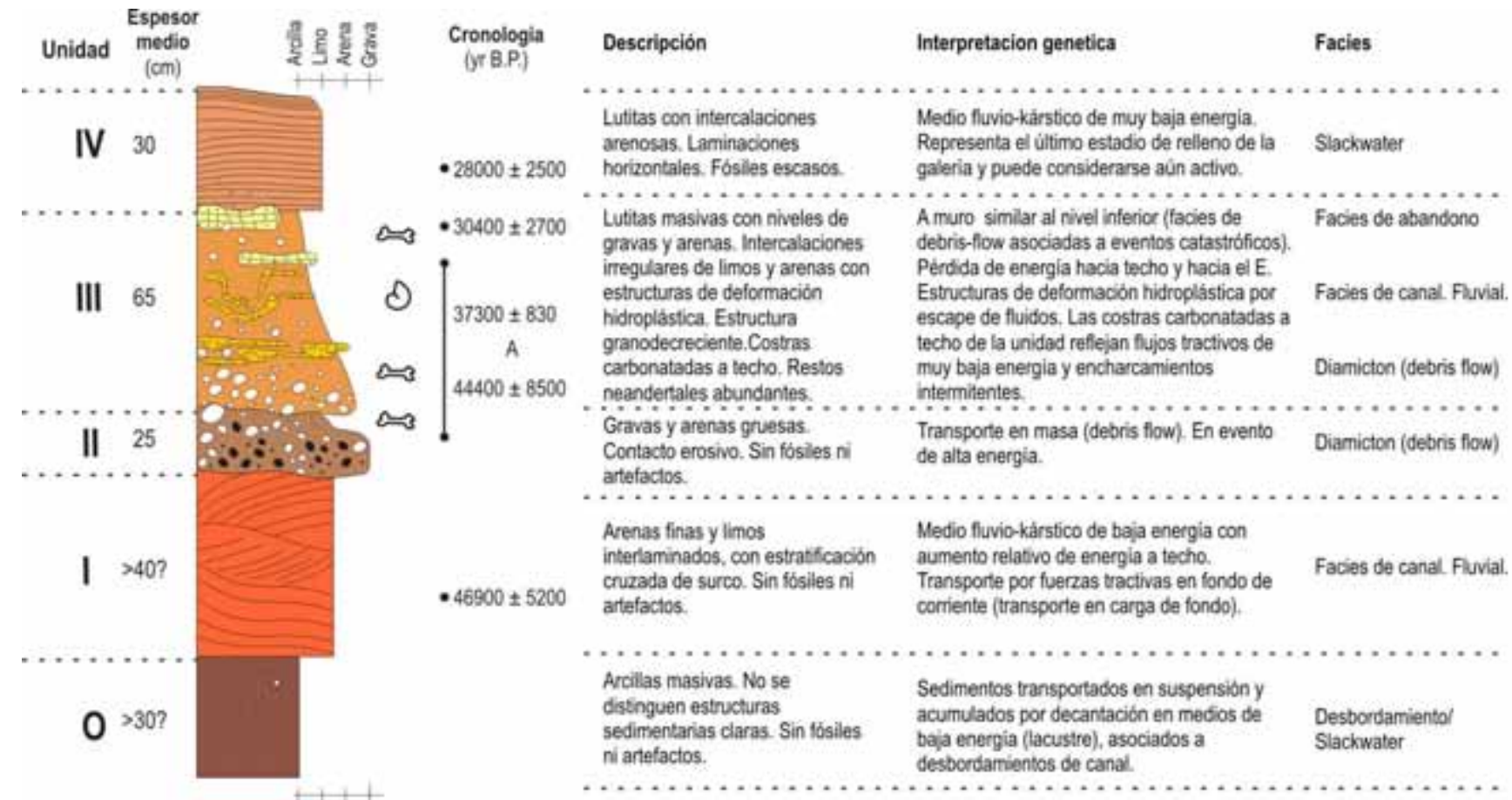

Figura 4. Columna litoestratigráfica tipo representativa del relleno de la zona central de la Galería del Osario; con descripción somera de las principales unidades sedimentarias definidas y su interpretación genética. Modificado de Cañaveras et al. (2011).

Figure 4. Stratigraphic column from the central zone of the Ossuary Gallery, with a brief description of the main sedimentary units and their genetic interpretation. Modified from Cañaveras et al. (2011).

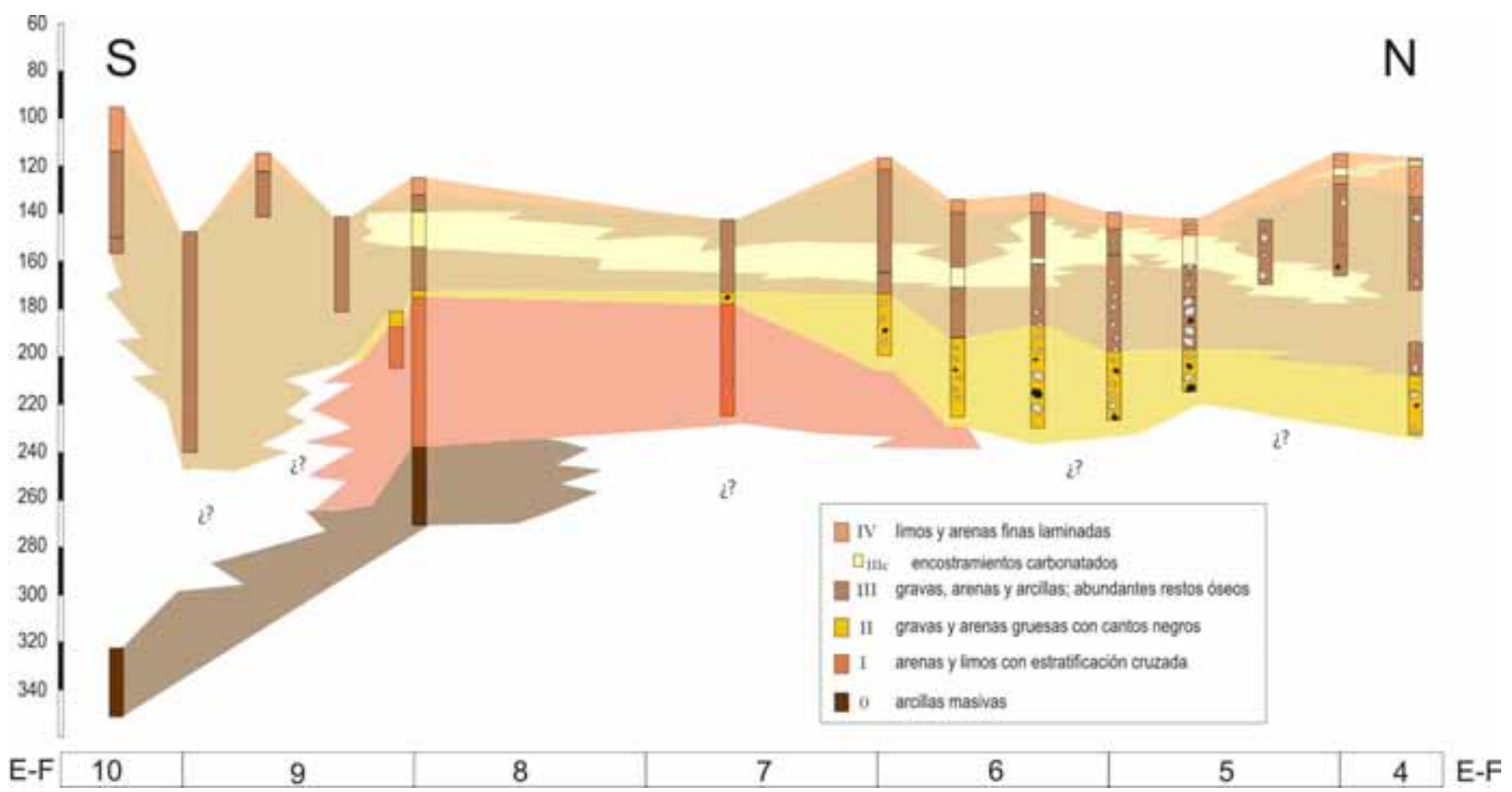

Figura 5. Correlación litoestratigráfica en E-F de los cortes levantados desde 2002 a 2013. Modificado de Cañaveras et al. (2011). Figure 5. Lithostratigraphic correlation in E-F (studied sections from 2002 to 2013). Modified from Cañaveras et al. (2011). 
Osario. Este nivel está asociado a la presencia de minerales ferromagnéticos $y$, en concreto, a la presencia de altos contenidos en elementos metálicos.

Unidad III: Esta unidad alberga la mayor parte de los restos arqueológicos y antropológicos encontrados en la cueva del Sidrón. Se trata de una unidad litoestratigráfica compleja, con gran variabilidad espacial, tanto en la horizontal como en la vertical. La estructura general de la unidad es granodecreciente (pérdida de energía a techo) pudiéndose distinguir tres términos con diferente desarrollo (tipología y espesor) según secciones o zonas: Illa) Tramo basal compuesto por gravas, arenas y lutitas mezcladas irregularmente que se asemeja a los materiales de la unidad anterior, pero sin el carácter grano-soportado dominante de la Unidad II. IIIb) Potente sucesión de lutitas masivas con intercalaciones irregulares de limos y arenas con estructuras de deformación hidroplástica (escape de fluidos). Illc) Niveles de encostramientos carbonáticos de espesor y textura variable que presentan una disposición horizontal y una alta continuidad lateral. Estos encostramientos alcanzan mayor desarrollo y espesor en hacia la pared este de la galería. Como se muestra en la figura 6 , se denota una cierta tendencia al incremento de la susceptibilidad magnética hacia techo en esta unidad, que coincide con la disminución progresiva del tamaño de grano y por tanto de la energía hidráulica.

Unidad IV: Está formada por limos y arcillas con intercalaciones arenosas. Presenta laminaciones subhorizontales bastante continuas. Representan el último estadio de relleno de la galería y puede considerarse aún activa. En las zonas más septentrionales de la galería, aguas abajo, esta unidad presenta peor selección e intercalaciones de finas $(0,5$ y $3 \mathrm{~mm})$ capas de carbonatos. Hacia el sur (interior de la galería) esta unidad presenta laminaciones más continuas y una pendiente deposicional hacia el E-NE. Se han encontrado restos óseos, aunque en una cantidad mucho menor a la de la unidad subyacente.

La geometría y espesor de estas unidades es variable no solo en la dirección del eje de la cavidad (Fig. 5) sino también en su perpendicular. Hacia el este de la galería, los depósitos son marcadamente más energéticos, como queda reflejado en el predominio de materiales gruesos en la unidad III y su carácter caótico y mal seleccionado (Fig. 7).

La asociación espacial de los restos humanos y líti-
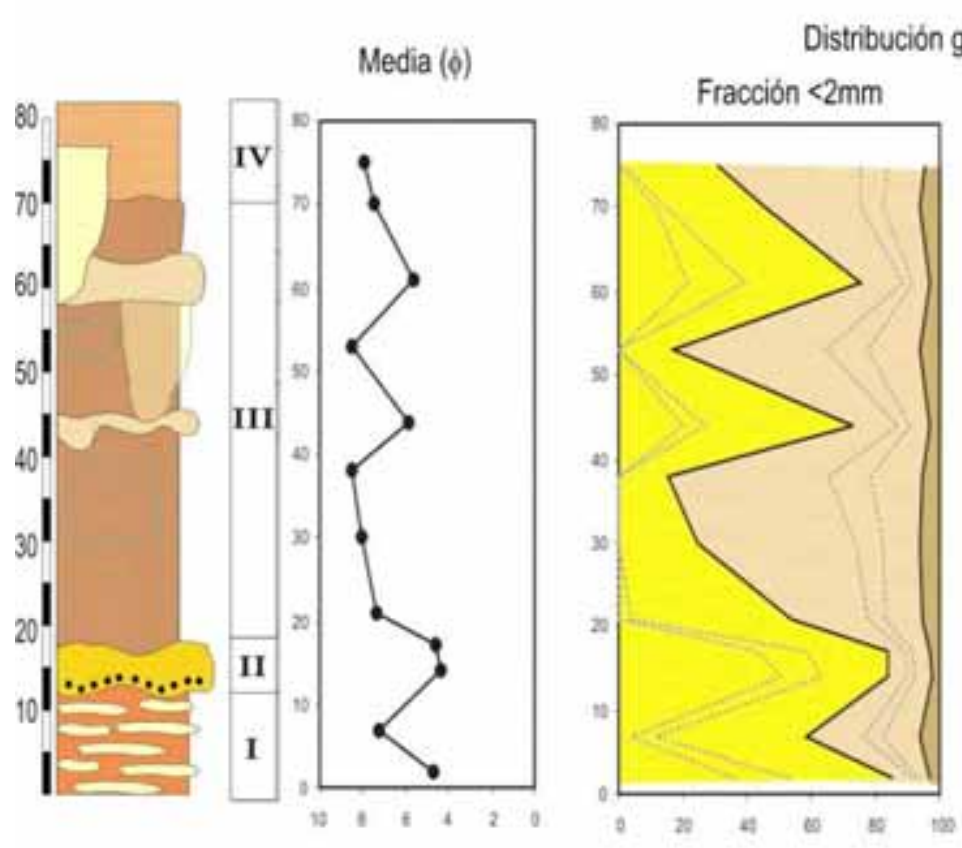

Distribucior
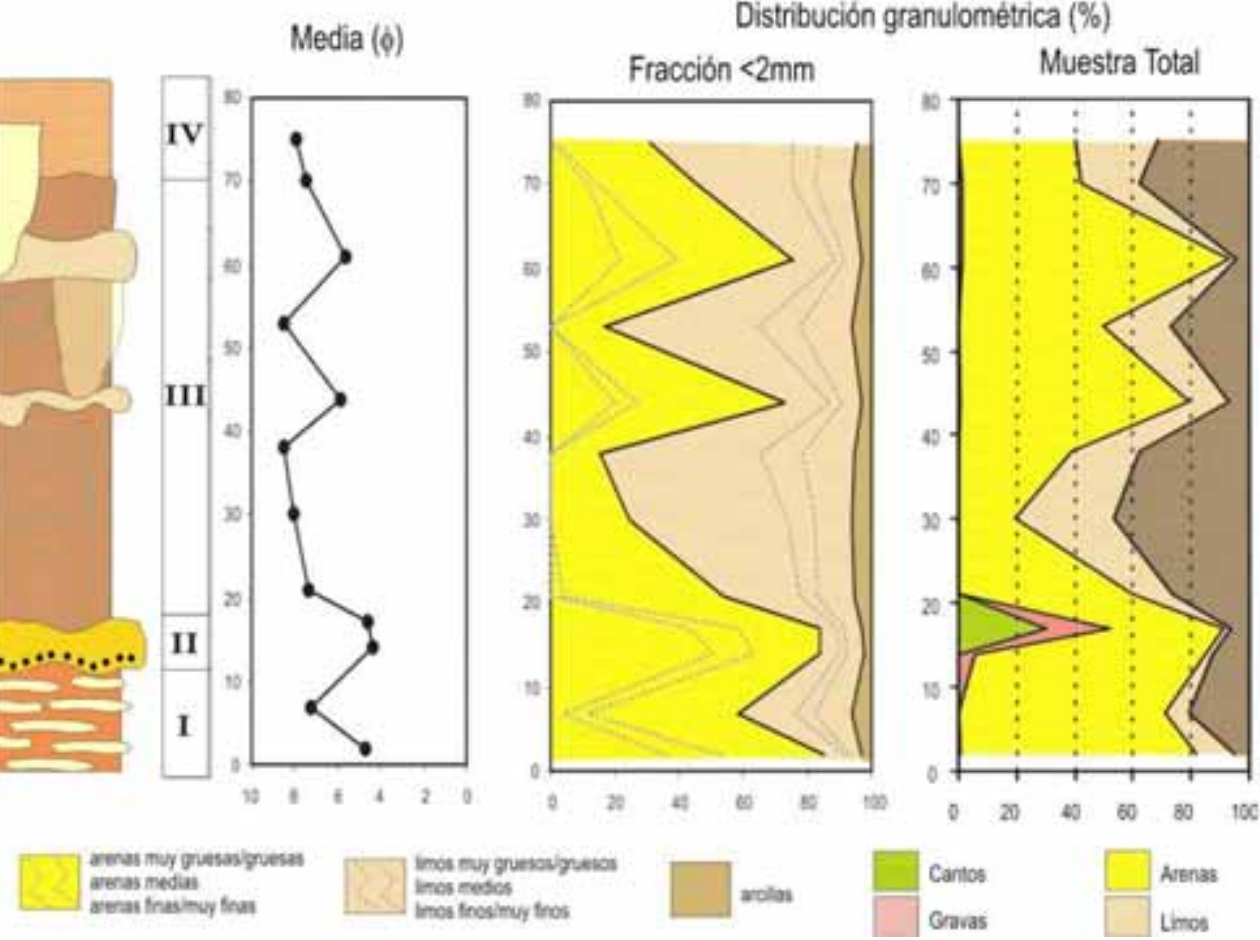
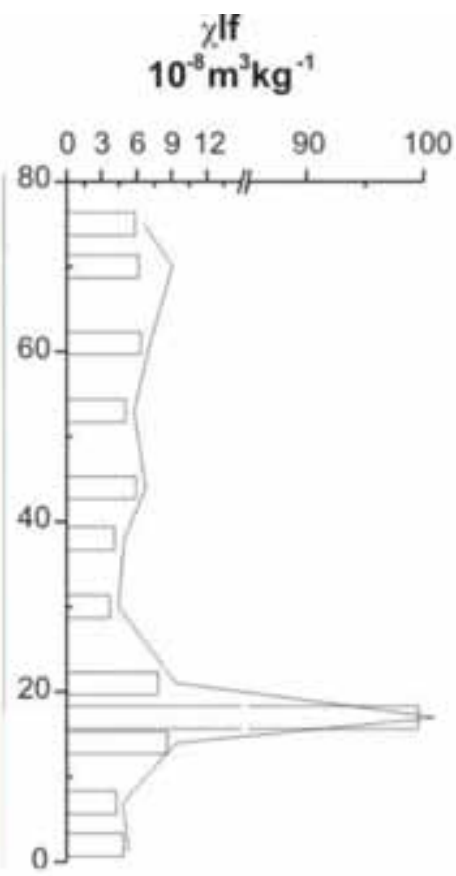

\section{Magnetismo ambiental}

Figura 6. Síntesis de los resultados de granulometría, composición y magnetismo ambiental del corte de la sección F-G / 8. Modificado de Cañaveras et al. (2011).

Figure 6. Summary of results of particle size, composition and environmental magnetism data from the stratigraphic section in F-G / 8 . Modified from Cañaveras et al. (2011). 


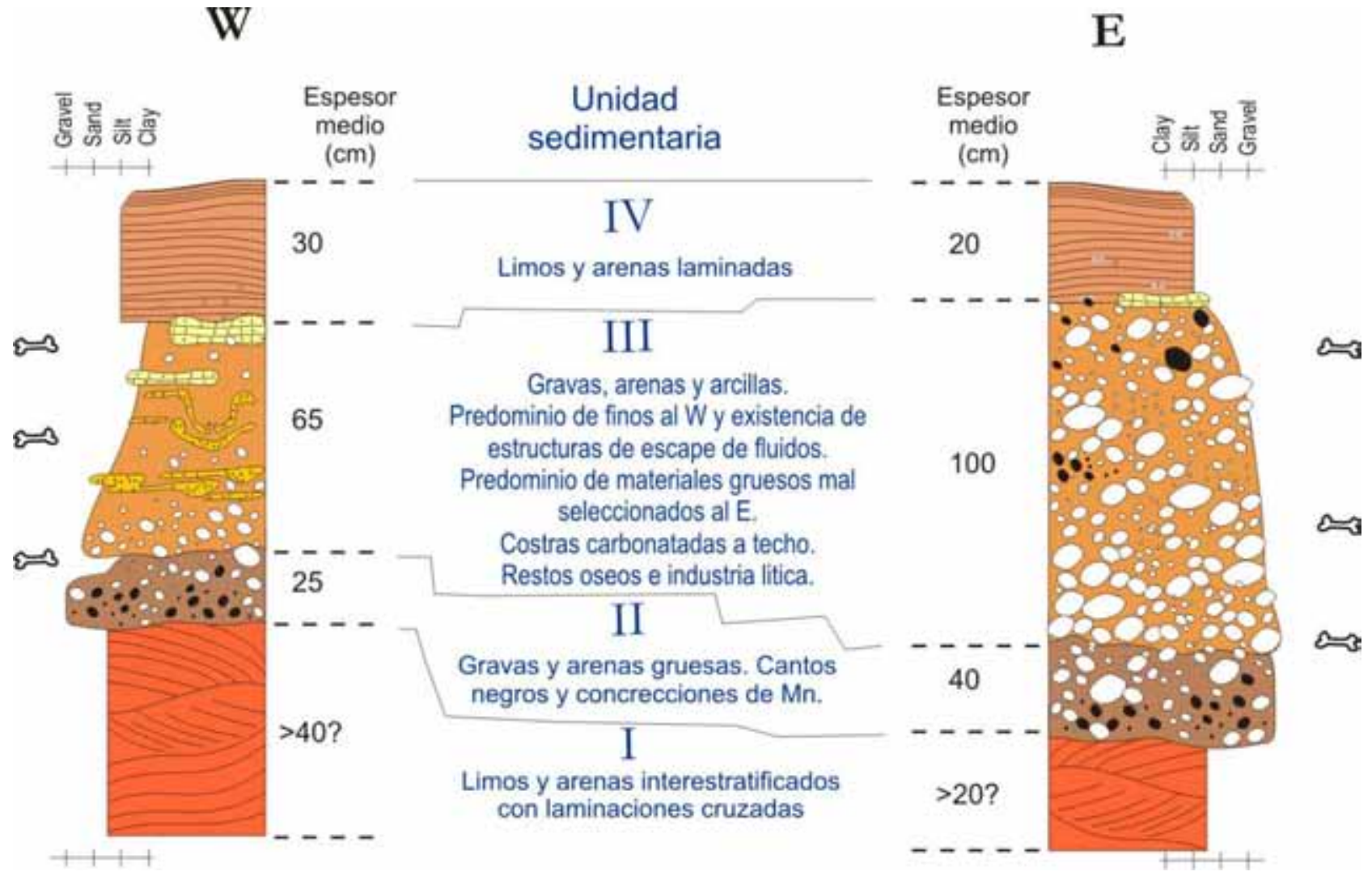

Figura 7. Columnas estratigráficas representativas de la zona central de la Galería del Osario, con descripción somera de las principales unidades sedimentarias definidas.

Figure 7. Representative lithostratigraphic columns from the central zone of the Ossuary Gallery, with a brief description of the main sedimentary units.

cos en esta zona es clara. Se localizan en una superficie aproximada de $\sim 12 \mathrm{~m}^{2}$, predominantemente en el seno de la Unidad III. Vistos en alzado (Fig. 8) los materiales buzan hacia el Este (corte transversal) y secundariamente hacia el Norte o hacia el Sur (corte longitudinal). Esta distribución, con una acusada pendiente hacia el este y algo más suave hacia el Norte o hacia el Sur, corrobora la posición secundaria (transportada) del material arqueológico (Ios materiales forman parte de un cono de deyección cuyo vértice se localiza en los cuadros $\mathrm{G} / \mathrm{H}-9 / 10$ ) e indica claramente la dirección de los aportes.

Al sur de esta zona central (cuadros F-G-H/13-1415; corte 7, ver Fig. 3B) se han reconocido los siguientes tramos o unidades, de muro a techo (Fig. 9):

II. Arenas heterométricas con pasadas centimétricas y discontinuas de limos y arcillas y niveles de gravas. Las gravas están mal seleccionadas y grano-soportadas, localmente componen niveles de espesor decimétrico y predominan a techo del tramo, donde también abundan cantos negros (con pátinas de óxidos de hierro y manganeso).
III. Arcillas y arcillas arenosas con algún canto de grava disperso. Hacia techo la granulometría se hace más fina y hacia el Oeste aparecen encostramientos carbonáticos de morfología irregular (IIIc).

IV. Limos y arcillas laminadas con cantos dispersos.

Los restos encontrados (algunas lascas y huesos de fauna) son muy escasos, pudiendo provenir de la zona meridional de la galería transportados previsiblemente por el agua.

Más al Sur de la Galería del Osario (Zona Corte 4 y 6 , ver Fig. 3B) la tipología de los materiales de relleno cambia con respecto a los anteriormente descritos, especialmente en lo que respecta al contenido en restos arqueológicos. En las cuadrículas F/20-21-22, se han reconocido dos grandes grupos de materiales, por un lado los gruesos (gravas heterométricas grano- o matriz-soportadas), entre los que pueden distinguir diversas subunidades en función de la naturaleza predominante de la matriz (arcillas, arenas, gravas finas), y por otro unos niveles de material fino, poco estructurado. Una fina capa de limos-are- 

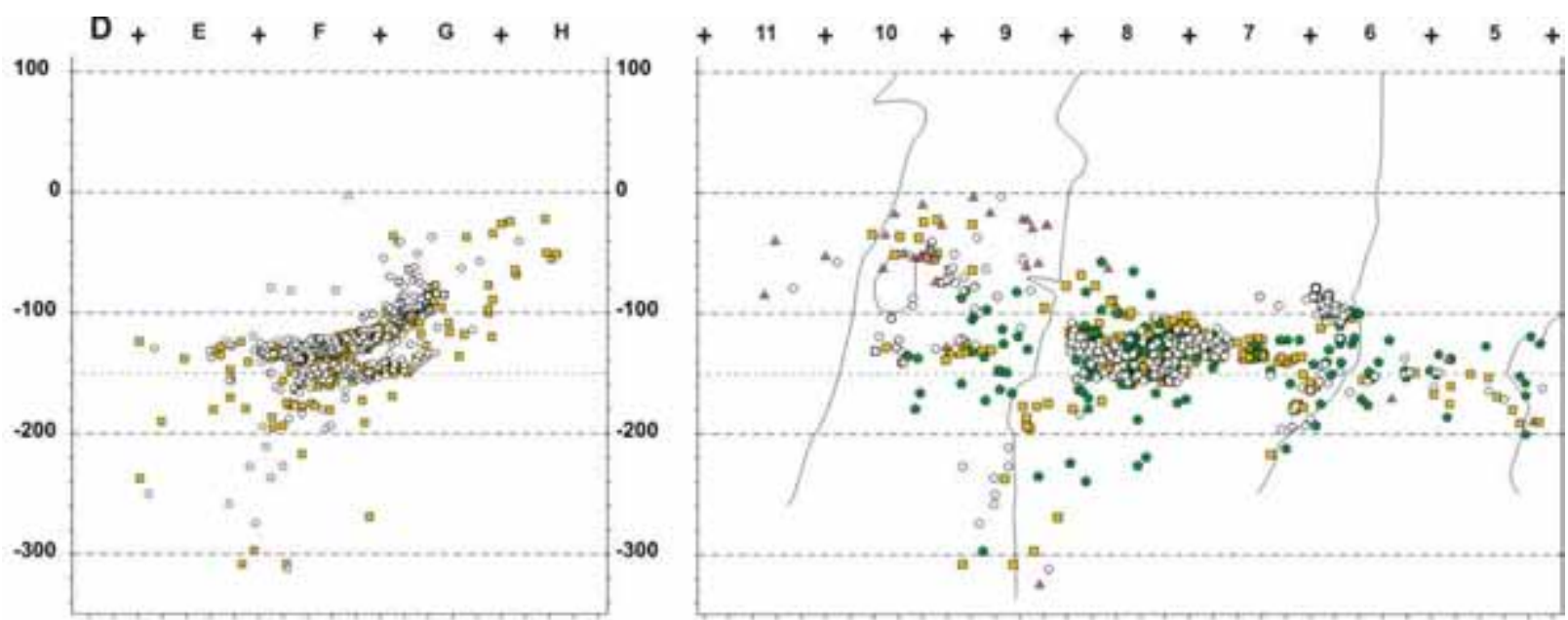

\section{Hueso humano $\square$ Hueso indet $\Delta$ Hueso fauna 1 Industria lítica}
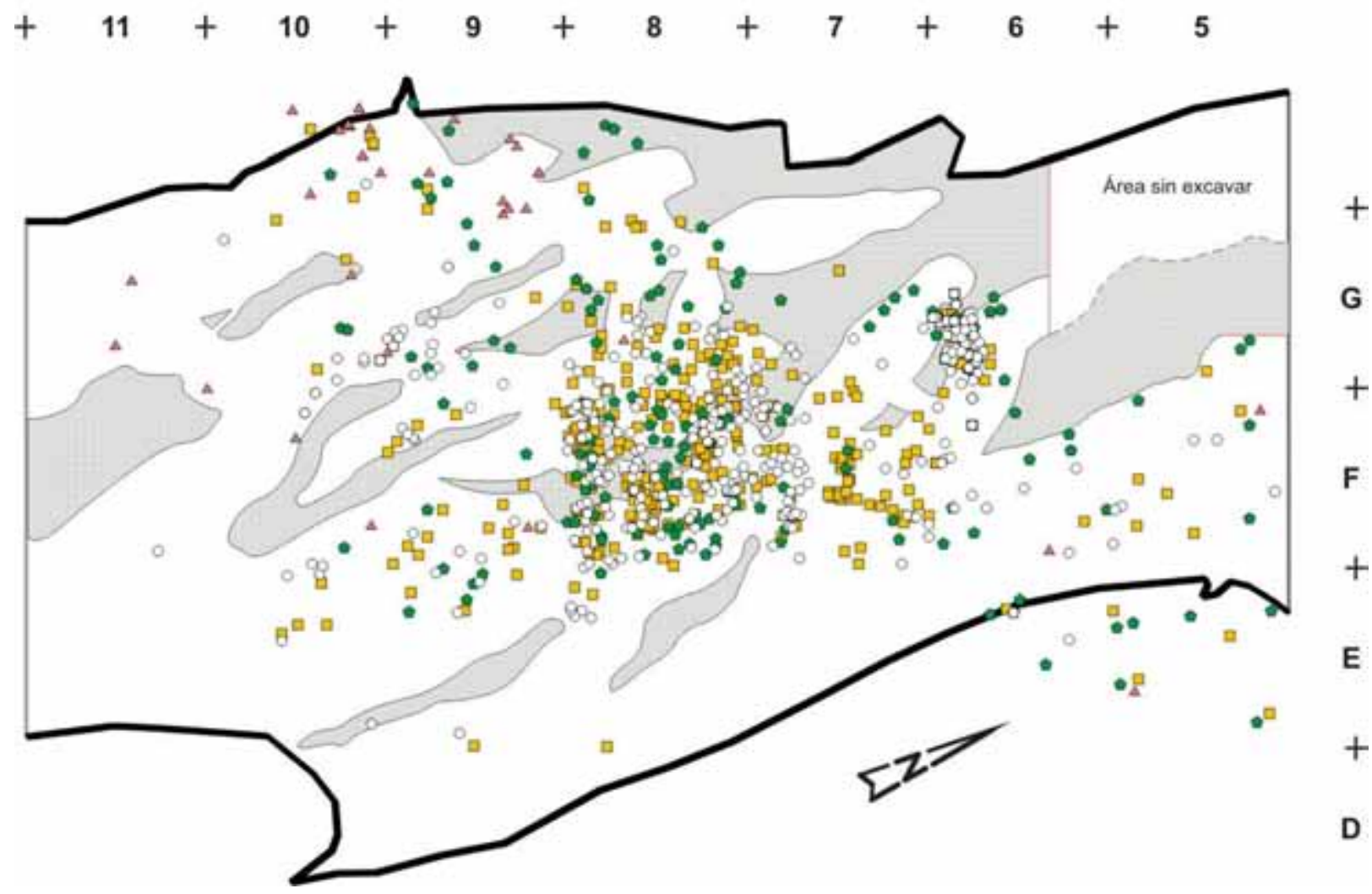

Figura 8. Distribución espacial del material arqueológico. Alzados: longitudinal (arriba izquierda) y transversal (arriba derecha) y vista en planta (abajo) siguiendo el eje norte-sur de la Galería del Osario (Santamaría, 2012).

Figure 8. Spatial distribution of archaeological material. Elevations: longitudinal (top left) and transverse (top right) and plan view (below) along the N-S axis of the Ossuary Gallery (Santamaría, 2012).

nas finas para-actuales cubre todo el corte. El paquete de gravas presenta una geometria cónica que marca una entrada o área de aporte diferente a los niveles de gravas ricos en restos óseos de la zona centro-septentrional de la galería. Los restos de macrofauna se concentran en los niveles groseros (a techo de lb y a muro de Ic). De muro a techo los tramos reconocidos en el paquete de gravas son (Fig. 10):

la. Gravas gruesas grano-soportadas, mal selec- 


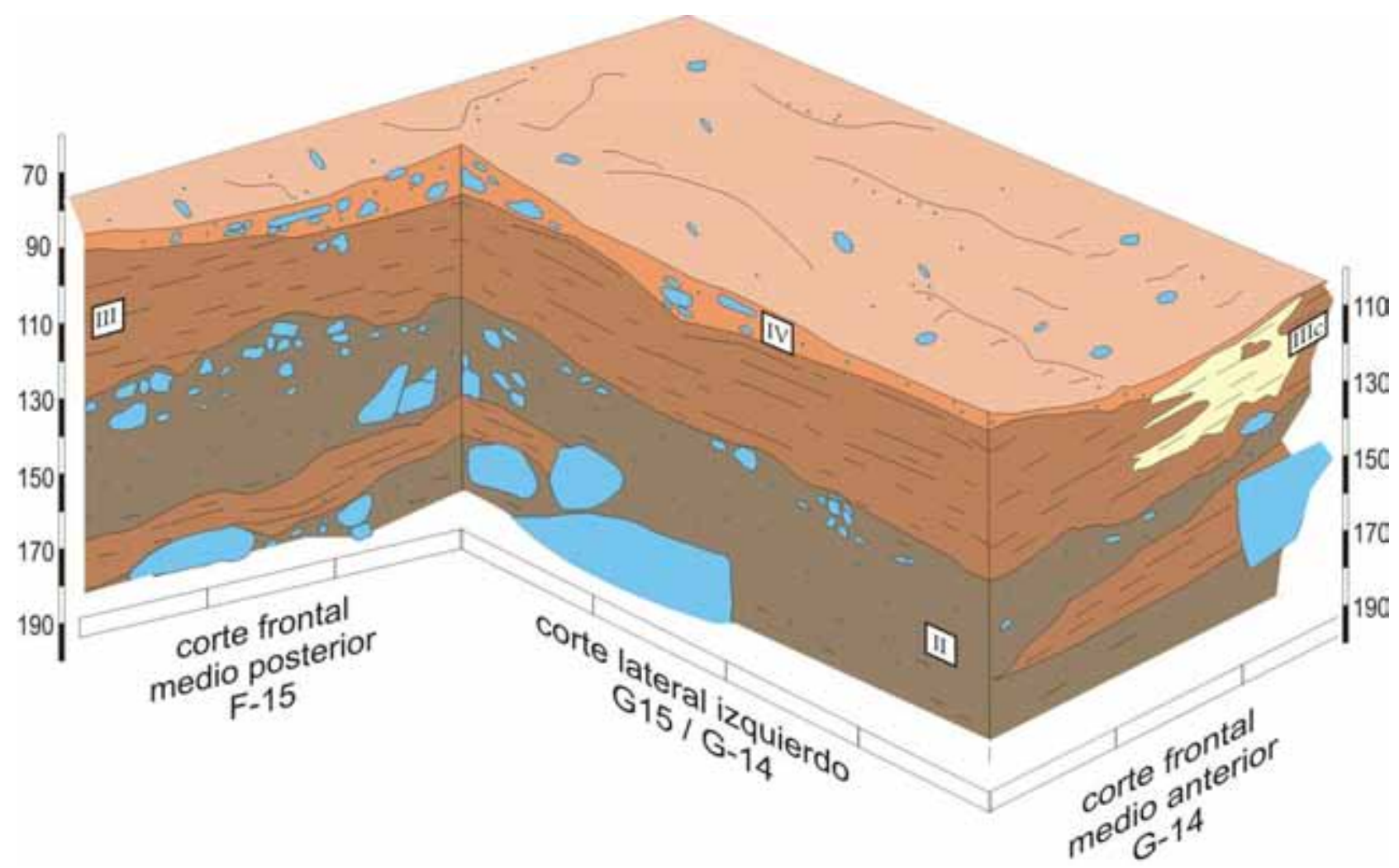

Figura 9. Secciones litoestratigráficas en el corte 7 (2014) (cuadrículas F-G/13-14-15).

Figure 9. Lithostratigraphic sections in section 7 (2014) (grids F-G / 13-14-15).

cionadas y polimícticas. La matriz es predominatemente arenosa (arenas medias a gránulos) y se va haciendo más arcillosa a techo, así como también aumenta el carácter matrizsoportado del conjunto. Abundan los cantos negros (con pátinas de óxidos de hierro y manganeso).

Ib. Gravas gruesas grano-soportadas a matrizsoportadas, mal seleccionadas y polimícticas (incluyen cantos negros) con una matriz arenosa/arcillosa que se va haciendo más fina a techo.

Ic. Gravas matriz-soportadas, mal seleccionadas. La matriz es arcillosa. Los cantos siguen siendo heterométricos como en los subniveles inferiores pero aquí son menos abundantes, incluidos los que presentan pátinas ennegrecidas.

En el extremo Sur de la galería (cuadrículas DE/21-22-23; corte 4, Fig. 3B) el relleno de la Galería (Fig. 11) presenta un tramo basal de granulometría fina (I), compuesto de arenas limo-arcillosas con cantos $(2 \mathrm{~mm}$ a $5 \mathrm{~cm})$, que pasa, en contacto erosivo a una capa $(60-100 \mathrm{~cm})$ de gravas gruesas grano-soportadas, polimícticas (calizas, cuarcitas...), mal seleccio- nadas, con matriz arcillosa-arenosa fina (III). Localmente, hacia el Norte, se apoya, también erosivamente, un paquete de gravas matriz-soportadas con cantos de hasta $4 \mathrm{~cm}$ y matriz arcillosa, algo limosa. A techo se sitúa un nivel de $20-30 \mathrm{~cm}$ de arenas finas y limos con cantos (III). En este nivel la granulometría se hace más fina a techo, culminando con una fina costra carbonatada (IIIc). Por ultimo, una fina sucesión de arenas finas-muy finas, laminadas, culmina la sección (IV).

En el extremo sureste de la galería (I/24-25-26; J$\mathrm{H} / 25$; Corte 1, Fig. 3B) se han distinguido 6 unidades sedimentarias en las que se discriminan dos episodios en el relleno separados por una costra estalagmítica (a techo del tercer tramo) (Fig. 11). De muro a techo:

- 50 a $55 \mathrm{~cm}$ de arcillas limo-arenosas pardas con intercalaciones de laminaciones arenosas claras y algunas acumulaciones de materia orgánica y/o nódulos de Mn. Hacia techo van pasando a arenas con algunas gravas en secuencia granocreciente.

- 5 a $15 \mathrm{~cm}$. de gravas grano-soportadas en relleno de canal erosivo sobre el paquete anterior. 

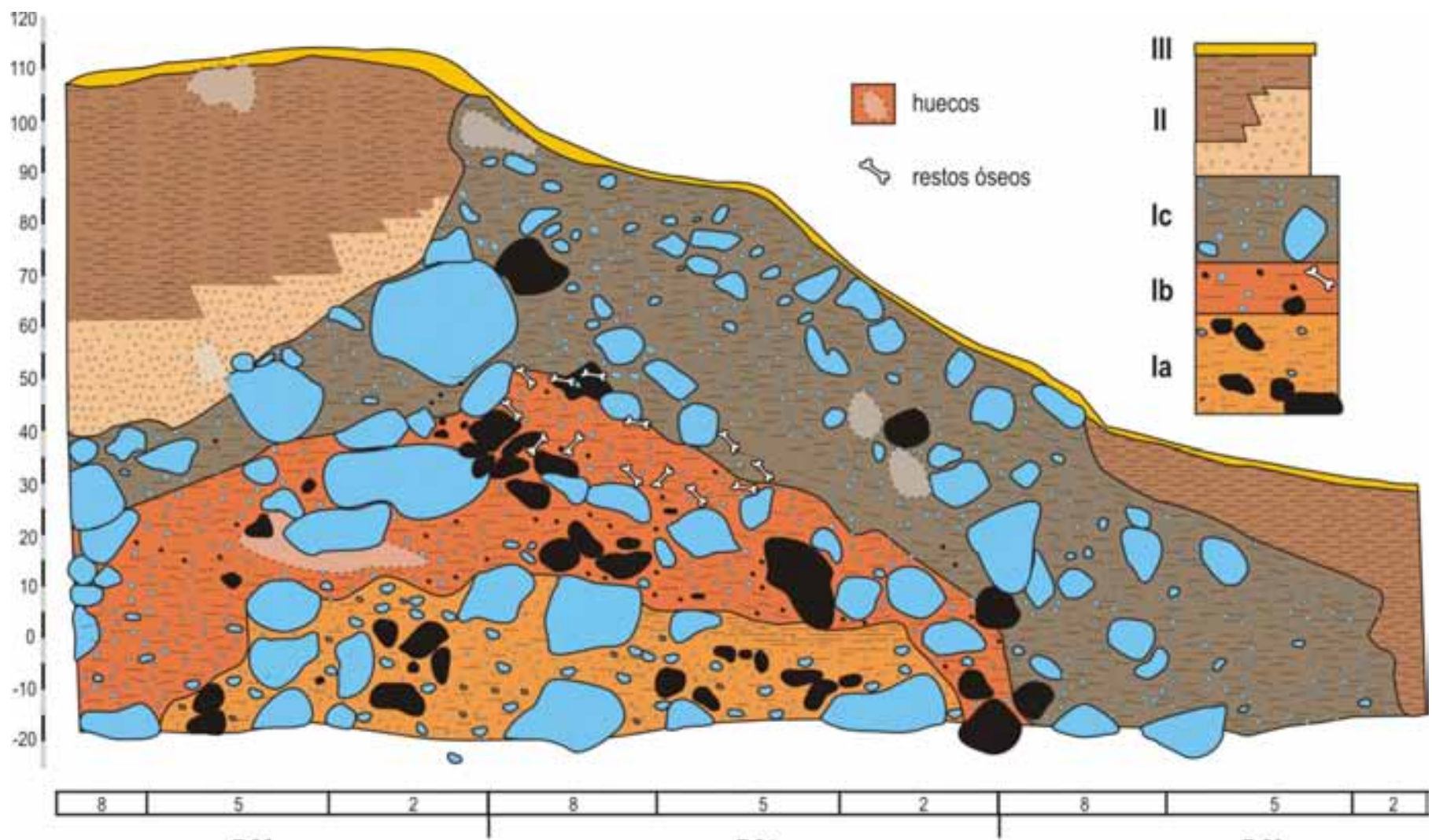

\section{F 22}

F 21

$\mathrm{F} 20$

Figura 10. Sección litoestratigráfica (cuadrículas F/20-21-/22) en el Corte 6

Figure 10. Lithostratigraphic section (grid F/20-21-22) in section 6.

Hacia techo incrementa el tamaño medio de las gravas (de 3 a $5 \mathrm{~cm}$ ). Las gravas pasan a un nivel arcilloso-limoso de tonos anaranjados a crema de $1-2 \mathrm{~cm}$.

- 60 a $70 \mathrm{~cm}$. de un depósito caótico de cantos principalmente carbonáticos que en la base presentan pátinas negras ricas en óxidos de $\mathrm{Mn}$. A techo aparece una fina colada estalagmítica centimétrica de composición mayoritariamente calcítica.

- 20 a $25 \mathrm{~cm}$. de arenas muy finas limo-arcillosas de tonos ocres que presentan laminación paralela y estratificación cruzada. Las arenas aparecen dispuesta en niveles centimétricos con intercalaciones de niveles de 1 a $3 \mathrm{~mm}$ de arenas blancas algo más gruesas y bien seleccionadas. Hacia techo van haciéndose progresivamente más finas en una secuencia granodecreciente.

- 10 a $13 \mathrm{~cm}$. de gravas grano-soportadas moderadamente seleccionadas y con matriz arenosa. Lateralmente pasan a arenas gruesas. Entre las gravas, con un tamaño medio de 2 a $3 \mathrm{~cm}$, aparece algún canto con pátina negra.

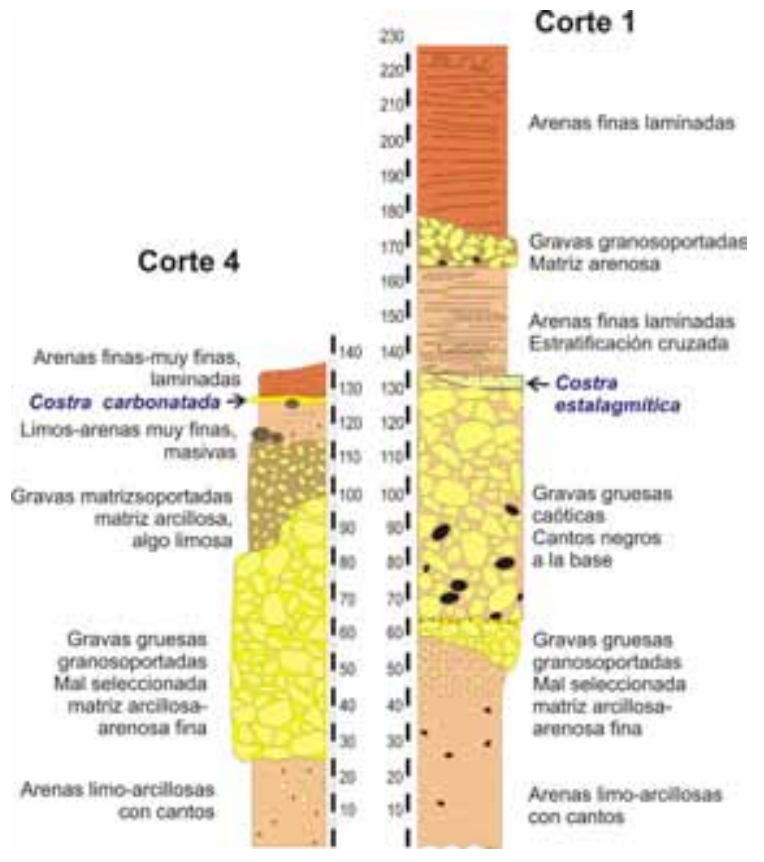

Figura 11. Columnas estratigráficas de los depósitos del fondo de la Galería del Osario.

Figure 11. Stratigraphic columns corresponding to southern infill deposits of the Ossuary Gallery. 
- Arenas limo-arcillosas a muro que progresivamente van pasando a limos y arcillas arenosas de color ocre. Presencia de laminaciones, localmente disturbadas (huellas de escape de fluidos).

La correlación con los sedimentos del centro de la galería no es completa. En este extremo se encuentran solo bien representados parte de los depósitos limo-arcillosos basales (Unidad I), de los de la unidad III y los del último estadio de relleno de la galería (Unidad IV).

En la repisa situada sobre las cuadrículas $\mathrm{H} / 11-12$ (Fig. 12) se reconoce un depósito espeleotémico consistente en una colada estalagmítica (flowstone). En ella se observa que existen varias etapas de forma-
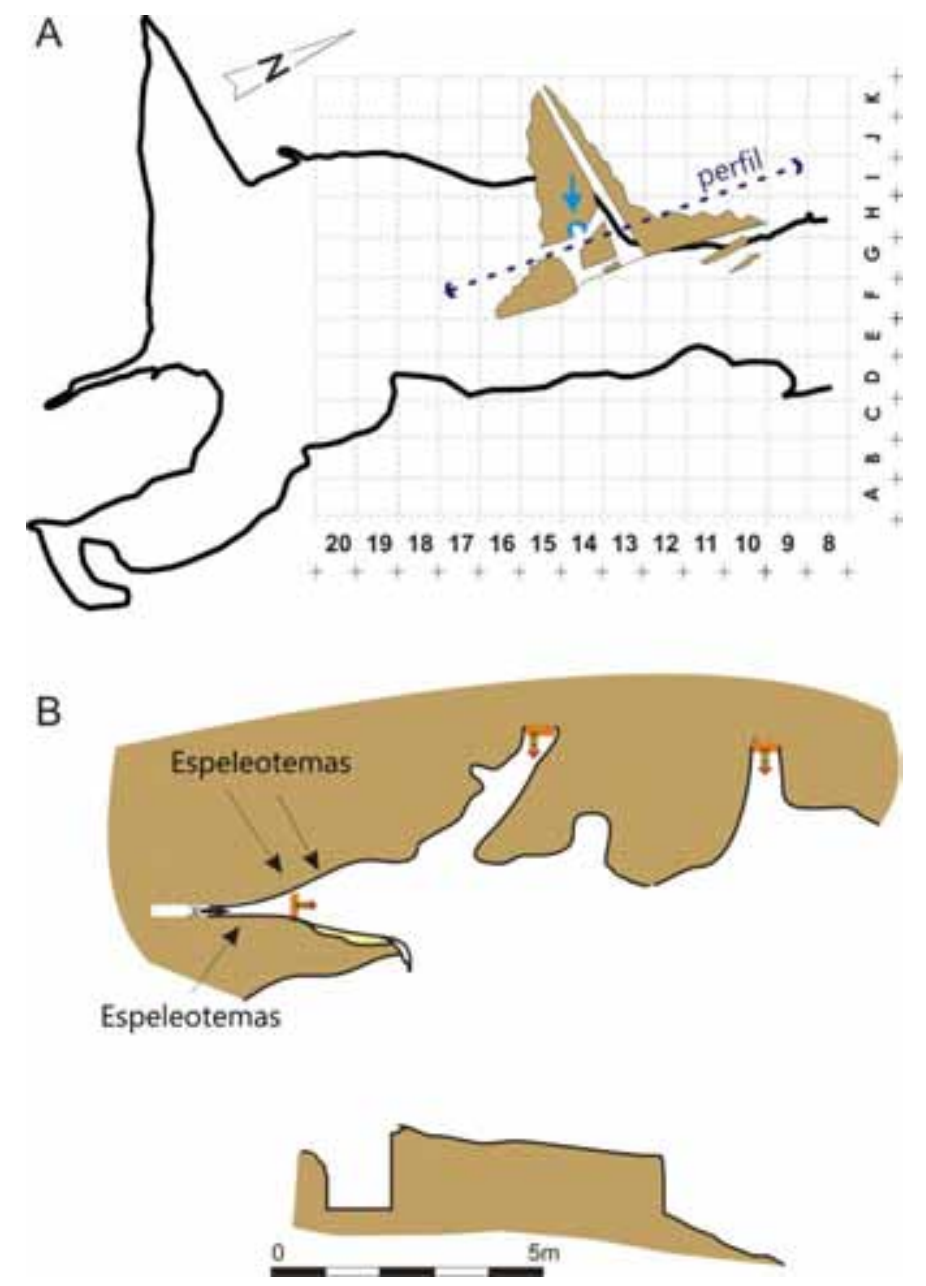

Figura 12. Conductos en el techo de la zona central de la Galería del Osario. A) Representación en planta con indicación en azul del conducto en $\mathrm{H}-14$. B) Perfil.

Figure 12. Conduits in the ceiling of the central area of the Ossuary Gallery. A) Representation plan with the conduit in $\mathrm{H}-14$ in blue. B) Profile sketch. ción para el depósito estalagmítico sobre y entre las cuales existe un relleno sedimentario arenoso que proviene de varios conductos abiertos en la pared Oeste de la Galería del Osario y que van a dar a dicha colada. Estos sedimentos detríticos son ricos en microfauna. Asimismo, es destacable que en alguno de los depósitos intercalados con las coladas aparecen gravas dispersas y entre ellas algunos cantos negros con tamaños de 5-8 $\mathrm{mm}$. Tras excavar las costras y extraer el depósito existente se ha localizado un conducto situado en la vertical de la cuadrícula $\mathrm{H}-14$ que tiene un diámetro irregular de $\sim 30 \mathrm{~cm}$ y un desarrollo vertical que alcanza una altura mínima, hasta donde se ha podido medir, de 1,63 $\mathrm{m}$.

\section{Zona Salomón - Galería Cimera}

La Galería Cimera forma parte del nivel superior de los cuatro niveles principales en los que se estructura el complejo kárstico de la cueva de El Sidrón (Alonso Peña, 2011; Silva et al., 2011). Se trata de un nivel situado a una cota de $172 \mathrm{~m}$ y cercano a la superficie ( 5 a $8 \mathrm{~m}$ en la vertical), en íntima conexión con otro nivel intermedio situado por encima del nivel de la Galería Principal. Dicha galería presenta una gatera hacia el norte denominada Pasu Salomón (Fig. 13) y un apéndice hacia el noreste en dirección a la Galería del Osario y, a su vez, en línea con el poljé.

En esta zona se ha excavado en varias áreas (Trinchera y Lengua, Fig. 13). El registro estratigráfico presenta similitudes con la secuencia de la Galería del Osario, si bien no está aun demostrado su equivalencia genética.

En la sección correpondiente a la zona más alta y meridional de la Galería Cimera (trinchera) y cerca del extremo sur del Pasu Salomón el depósito presenta dos grandes paquetes de sedimentos, de muro a techo (Fig. 14): III) Arenas y arcillas con grandes cantos, con intercalaciones de arcillas masimas laminadas. IV) Arenas laminadas de grano medio, con intercalaciones de paquetes de espesor centimético de arenas gruesas y gravas.

A lo largo de todo el corte son abundantes los encostramientos en forma de paquetes carbonatados (flowstones arenosos) de diverso espesor. Se hacen más abundantes hacia techo del corte, afectando al nivel superior.

En la zona de la Galería Cimera, al oeste del Pasu Salomón (Fig. 13) se han realizado también labores de excavación, concretamente en el conducto más oriental (Lengua) hacia la situación de la Galería de Osario. En el corte levantado se han reconocido los siguientes conjutos o unidades de sedimentos (de 


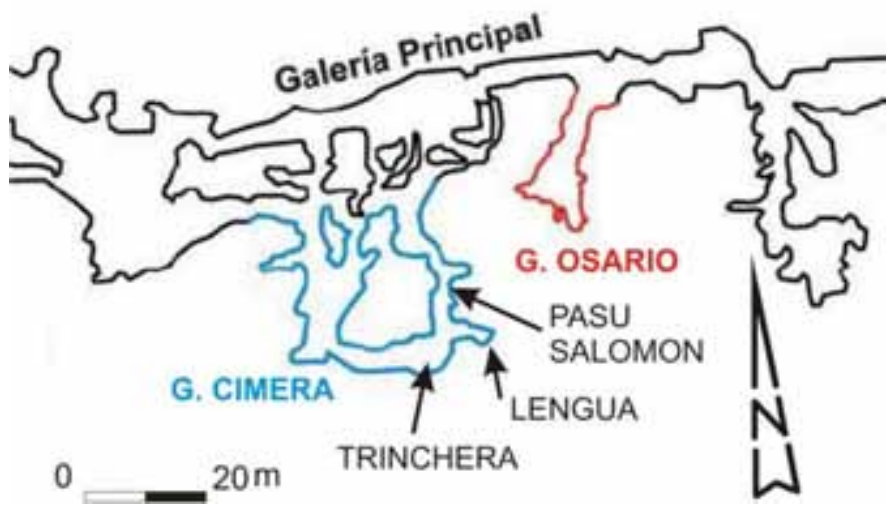

Figura 13. Situación de las áreas de excavación en Zona SalomónGalería Cimera.

Figure 13. Location of the excavation areas in the Salomón-Galería Cimera zone.

muro a techo) (Fig. 14): III) Arenas con grandes con cantos que pasan a techo a arcillas mas o menos laminadas con algunas gravas. IV) Arcillas con encostramientos calcáreos y huellas de raíces. A lo largo de todo el corte son abundantes los encostramientos en forma de paquetes carbonatados (flowstones arenosos) de diverso espesor. Se hacen más abundantes hacia techo del corte, delimitando el nivel superior (arcillas con encostramientos y huellas de raíces). En 2014 se profundizó en esta zona hasta una cota de $167,5 \mathrm{~m}$. El sedimento está compuesto por arcillas y cantos heterométricos que llegan a alcanzar los $30 \mathrm{~cm}$ de diámetro. Presenta escasos fragmentos óseos faunísticos, muy alterados y en curso de estudio. En este punto la roca madre se va estrechando en forma de grieta en dirección hacia el noreste, constituyendo una hipotética zona de entrada de los restos arqueológicos y antropológicos hacia la Galería del Osario a través de galerías intermedias.

\section{Interpretación y modelo de relleno}

El análisis sedimentológico detallado de la Galería del Osario viene a corroborar que los ambientes kársticos se caracterizan por una alta variedad y complejidad en su relleno sedimentario, tanto detrítico como químico (espeleotémico). El registro sedimentario de la Galería del Osario está incompleto y presenta bruscos cambios laterales de facies y discontinuidades.

Aunque se han reconocido procesos de sedimentación autóctona en el fondo de la Galería de Osario (colapso interno de techos o paredes), los sedimentos detríticos de la galería tienen un origen mayoritariamente alóctono, provienen del área de captación o cuenca hidrográfica (Depresión o polje de El Sidrón,
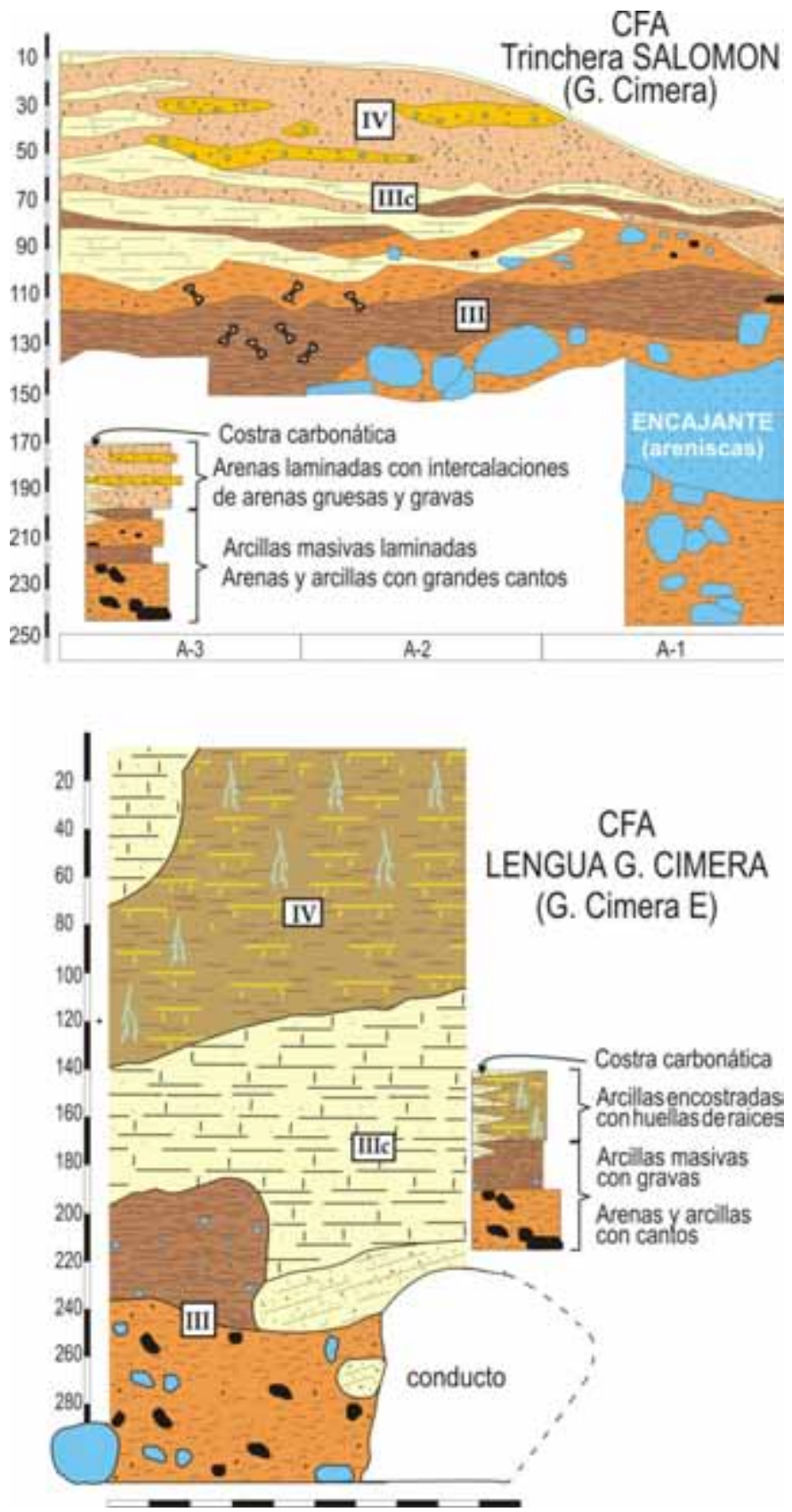

Figura 14. Secciones litoestratigráficas en Trinchera Salomón (2012) y en Lengua - Galería Cimera / Salomón (2013).

Figure 14. Lithostratigraphic sections in the Trinchera Salomón (2012) and Lengua - Galería Cimera / Salomón (2013).

desde el Pleistoceno) y han sido transportados a la cueva predominantemente por agua de forma episódica y como resultado de eventos de alta energía. Si bien el material transportado a la galería puede haber formado parte de otros depósitos superficiales formados previamente (terrazas, llanuras de inundación,...). 
La particular abundancia y distribución de los huesos e industria lítica en la Galería del Osario, y la ubicación de ésta dentro del sistema kárstico de la cueva de El Sidrón ha determinado que la elucidación del origen de éstos haya sido el objetivo principal del estudio geoarqueológico del relleno de la Galería del Osario. La caracterización y correlación de los perfiles litoestratigráficos estudiados en la Galería del Osario nos han permitido definir diferentes episodios que reflejan los cambios en la dinámica y en la procedencia del relleno de la cavidad (Figs. 4 y 15).

Los depósitos más antiguos, localizados en la zona central de la galería (Unidad 0), donde se concentra el material antropológico y arqueológico se formaron por la sedimentación de material fino en un cuerpo de agua estancado (facies de encharcamiento), y corresponden a las facies de menor energía del registro sedimentario de la galería. Le sucede un depósito fluvio-kárstico en facies de canal (Unidad I), que solo se halla preservado en las áreas más subsidentes y protegidas. Posteriormente sobrevienen episodios altamente energéticos (Unidades II y III basal) correspondientes a coladas de detritos (debris flow). El desarrollo (distribución y espesor) de estos depósitos es desigual en la galería. En la parte más occidental de la misma un evento de alta energía queda representado por el nivel de gravas mal seleccionadas

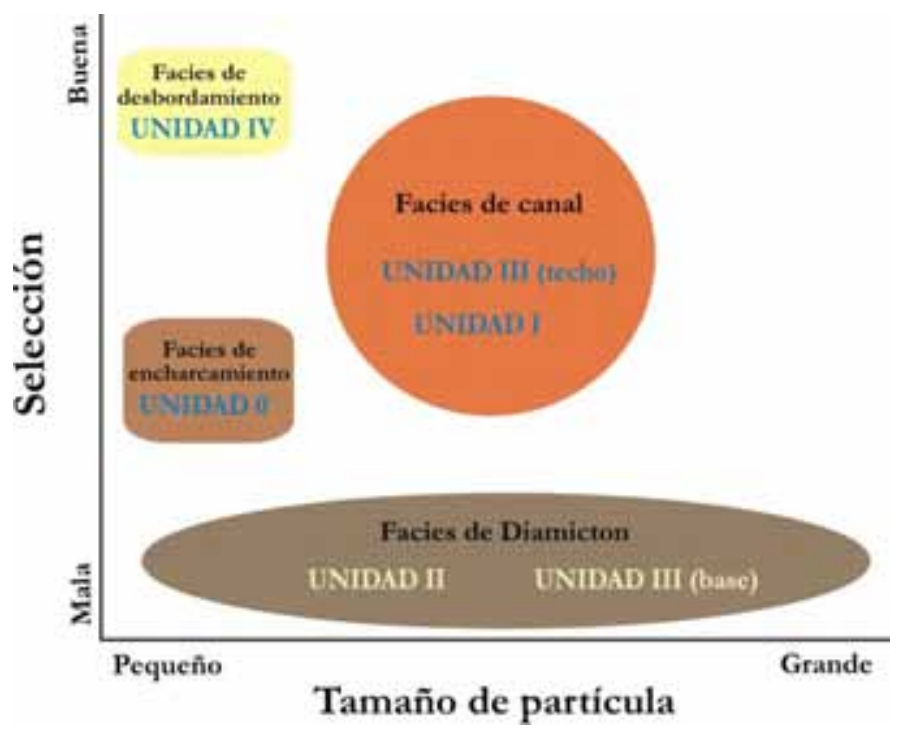

Figura 15. Esquema que ilustra los tipos de facies reconocidos en los sedimentos de la Galería del Osario en función de tamaño de partícula y del grado de selección del sedimento (basada en Bosh y White, 2004).

Figure 15. Diagram illustrating sediment facies identified in the at Ossuary Gallery in terms of sorting and particle size (based on Bosh and White 2004).
(Unidad II) con neto carácter erosivo respecto a la Unidad I, y la existencia de zonas de subsidencia o incluso un sumidero interno de la Galería hacia niveles inferiores del karst en la zona E-9/F-9 queda marcado por el gran espesor de la Unidad III en esta zona. Esta unidad es bastante compleja en cuanto a tipología y geometría de los sedimentos que la componen, aunque, hacia la zona occidental de la galería muestra una disminución progresiva de la energía hacia techo y el desarrollo de precipitados carbonatados (costras, coladas) en sus niveles superiores. Estos depósitos carbonatados son localmente complejos, abarcando desde términos micríticos laminados con más o menos terrígenos a coladas espeleotémicas (flowstone) constituidas por empalizadas de cristales columnares. En casi todos los casos están relacionados con aguas de flujo laminar circulado libremente una vez se ha cementado el sedimento sobre el que circula. El depósito de la Unidad IV vuelve a marcar un episodio de baja energía en la cavidad. Representa el último estadio de relleno de la galería y es todavía activo. Este tipo de depósitos formados por materiales finos y laminados (flujos fluviales de baja energía o facies de desbordamiento) constituye habitualmente el techo de la secuencia sedimentaria en otras partes de la cueva de El Sidrón y en la mayoría de las cuevas (Bosh y White 2004; Ford y Williams, 2007).

En la mitad sur de la Galería del Osario también se alternan los depósitos de alta y baja energía. En el extremo sureste de la galería (I/24-25-26; J-H/25) se distinguen bien dos episodios de relleno separados por una costra espeleotémica, uno inferior de alta energía compuesto predominantemente por gravas grano-sostenidas mal seleccionadas sin ninguna estructuración interna; y uno superior predominantemente arenoso con laminaciones e intercalaciones de gravas. El primero corresponde a un depósito en masa tipo coluvial o de debris flow; el segundo es un relleno también en pendiente pero mucho menos competente con predomino de depósitos fluvio-kársticos.

La concentración de los restos y la propia geometría de los depósitos que los contienen apuntan hacia un área de procedencia muy localizada en niveles superiores de la galería, hacia la zona septentrional a sur-occidental de la misma. Los huesos llegan a la Galería del Osario inmersos en un depósito (colada de barro/lodo y de detritos) de alta densidad y alta viscosidad que hace que la fricción entre clastos, incluidos los frágiles huesos, sea mínima, favoreciéndose un alto grado de conservación durante el transporte, estando algunos incluso en conexión anatómica. Además del importante porcentaje de piezas líticas que remontan (Santamaría, 2012; Santamaría 
et al., 2010; Santamaría et al., 2011). Este depósito en masa es el resultado de un evento catastrófico provocado por una fuerte avenida (inundación) producida presumiblemente por una gran tormenta. Este episodio de alta energía pone en actividad hidrodinámica una zona no activa en ese momento, ya fuera una entrada o un nivel superior al de la Galería del Osario (acumulación o almacenamiento intermedio). El análisis de las costras adheridas a los huesos muestra la estancia de algunos de los ejemplares en ambiente superficial (pátinas eólicas, rasgos de iluviación-eluviación, encostramientos biogénicos superficiales,...) indicando que estos provienen del exterior, aunque debieron estar en un ambiente protegido (e.g.: un abrigo o la zona de entrada de una galería) y su permanencia en condiciones superficiales debió ser corta, dadas las escasas huellas de alteración que presentan las piezas (Cañaveras et al., 2011).

Este almacenamiento intermedio, al igual que el emplazamiento más superficial (abrigo o entrada a una galería) se sitúa en un contexto totalmente vadoso, y se encuentran alejados y desconectados de la zona hidrodinámicamente activa del sistema kárstico. Actualmente esa entrada estaría tapada por depósitos coluviales y suelos sobre los que se desarrolla el bosque actual.

La morfología del conducto (anchura, sinuosidad, etc.) puede influir en las fluctuaciones del nivel del agua en episodios de crecidas y dar lugar a pronunciados gradientes energéticos a lo largo de la cueva que se reflejan en diferentes facies sedimentarias. Asimismo, episodios de alta energía pueden modificar e, incluso, eliminar total o parcialmente el sedimento depositado por un evento anterior. La especial configuración del fondo de la galería en tabiques y represas, ha sido responsable de la compleja geometría del relleno de la galería, pero, a su vez, ha favorecido la preservación de los restos óseos. Muchos de estos depósitos fosilíferos han quedado así colgados y protegidos de episodios de retrabajamiento y destrucción del registro sedimentario, tan habituales en la dinámica kárstica.

Con respecto al relleno estudiado en la Zona Salomón - Galería Cimera, los depósitos inferiores (unidad III) marcan la existencia de eventos de alta energía (gravas heterométricas de gran tamaño) sellados por depósitos de bajada del caudal o punta de crecida (arcillas laminadas). Los materiales de la unidad IV marcan un funcionamiento fluvio-kárstico normal, con deposito de arenas finas y media laminadas, con la existencia de algunos episodios (canales) más energéticos. La naturaleza y orientación de los encostramientos (hacia el interior de la galería) marcan el predominio de etapas de baja o nula sedi- mentación detrítica y/o de aportes siliciclásticos desde el exterior. En conjunto, esta zona pudo funcionar en un principio como colector del sistema fluviokárstico externo en un régimen hidrodinámico de energía variable y posteriormente, solo en eventos de alta energía, en comunicación con otros niveles inferiores del sistema kárstico, y llegando a producirse el cierre natural de la galería en su zona exterior.

La Galería Cimera forma parte del nivel superior de los cuatro en los que se estructura el complejo kárstico de la cueva del Sidrón. Se trata de un nivel cercano a la superficie (5-8 metros en la vertical), en intima conexión con un nivel intermedio formado por un complejo sistema de galerías. La Galería del Osario, junto a la Galería Principal correspondería a un nivel inferior. Estos niveles se relacionan genéticamente con niveles morfológicos exteriores (terrazas, fondos de valles, superficies de erosión...) que señalan la evolución del encajamiento de la red fluvial exokárstica, (Fig. 16): fondo actual del polje (160 $\mathrm{m})$, superficie asociada a encajamiento del sistema kárstico (164 m), nivel del polje (167-169 $\mathrm{m}$ ) y superficie superior $(180 \mathrm{~m})$.

\section{Conclusiones}

La Galería del Osario presenta una alta complejidad en su relleno sedimentario reflejo de la naturaleza episódica de su funcionamiento hidrodinámico y sedimentológico, de la alta energía de alguno de estos episodios, que queda reflejada en las características texturales y composicionales de los depósitos, así como en la geometría del propio depósito, y la irregular geometría del conducto, que compartimenta significativamente las áreas de deposición. La importante, por número y naturaleza, colección de restos arqueológicos y antropológicos se distribuye por una zona restringida de la Galería del Osario, y accedió a la misma en un episodio altamente energético, relacionado con eventos de inundación y/o tormenta. A la luz de estos datos se puede mantener la hipótesis de trabajo sobre el área fuente del material antropológico depositado la Galería del Osario (Cañaveras et al., 2011). Esta área fuente se sitúa en cotas superiores a la mencionada galería, es decir, en los niveles intermedio o superior, y el depósito o entrada de material óseo a la Galería del Osario se activaría en episodios de alta energía (crecidas, inundaciones). La aplicación metódica e integrada de métodos y técnicas geológicas (sedimentología, geomorfología, mineralogía, geofísica, etc.) ha resultado clave para resolver la problemática paleoambiental del registro antropológico y arqueológico de la cavidad. 

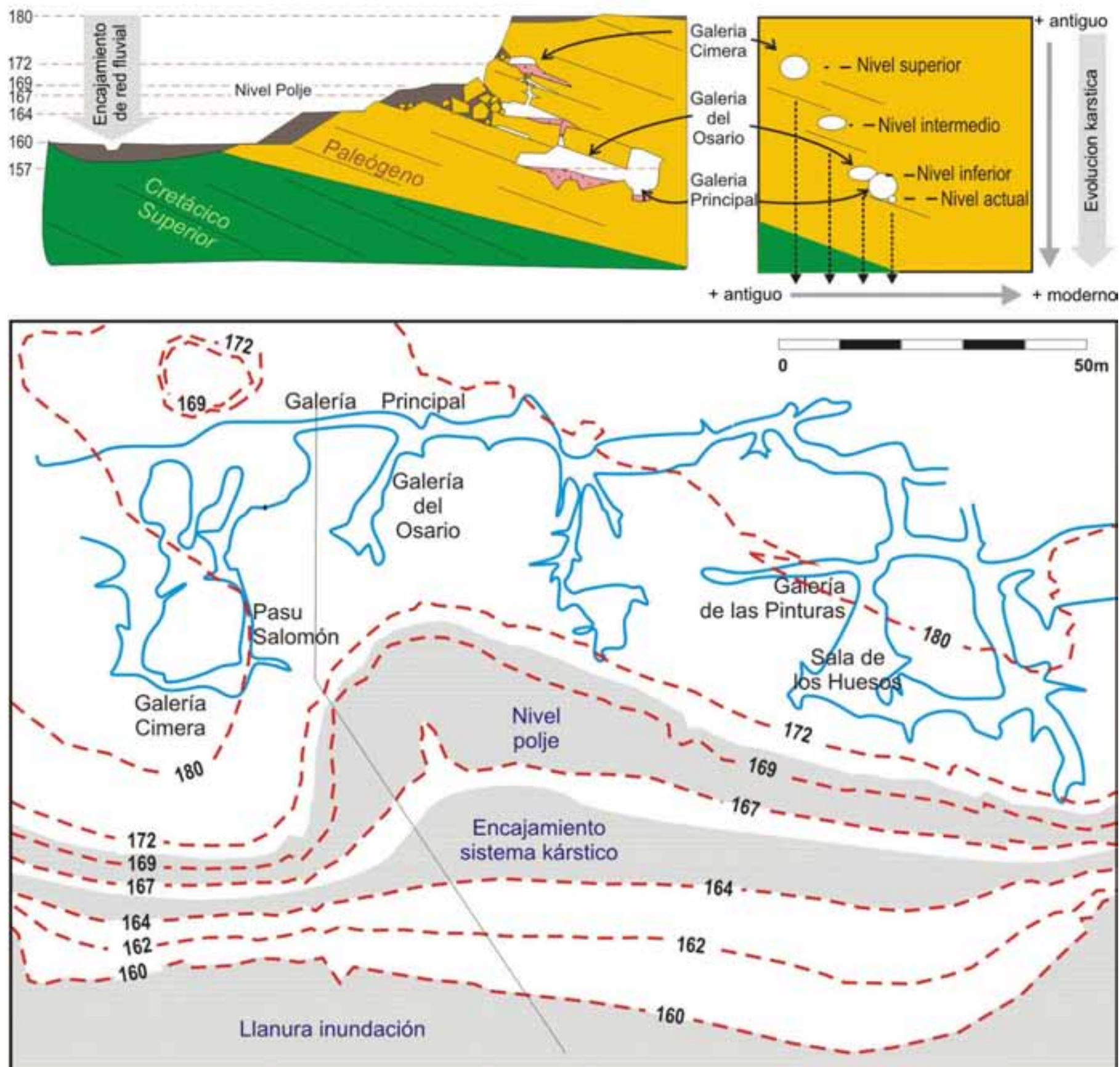

Figura 16. Correlación endokarst-exokarst y encuadre evolutivo de los niveles de cavidades del sistema kárstico de la cueva del Sidrón. Línea azul: contorno cavidades. Línea roja: curvas de nivel (m.s.n.m). Areas en gris: rellanos).

Figure 16. Endokarst vs. exokarst feature correlation and evolutive stages in the Sidrón cave karst system. Blue line: cave outline. Red line: topographic contour (m.a.s.l). Grey area: flat surface.

\section{Agradecimientos}

Este trabajo se ha financiado desde 1999 mediante diferentes contratos de investigación realizados entre el Gobierno del Principado de Asturias, la Universidad de Oviedo, la Universidad de Alicante, el
Museo Nacional de Ciencias Naturales (CSIC, Madrid) y la Universidad de Salamanca. Queremos agradecer la inestimable ayuda que nos han brindado durante todos estos años el magnífico equipo de excavadores que paciente y concienzudamente nos ha hecho el trabajo infinitamente más fácil. Un recuerdo especial 
a nuestros queridos Javier Fortea (1946-2009), sin el que este trabajo distará mucho de ser perfecto y Manolo Hoyos (1944-1999), maestro geólogo y pionero geoarqueólogo.

\section{Referencias}

Alonso Peña, J. 2011. Descripción física del complejo cárstico y sus conexiones exteriores. In: Rasilla, M., Rosas, A., Cañaveras, J.C. y Lalueza-Fox, C. (eds.), La cueva de EI Sidrón (Borines, Piloña, Asturias). Investigación interdisciplinar de un grupo neandertal. Excavaciones Arqueológicas en Asturias, Monografías I, Consejería de Cultura y Turismo. Oviedo, 21-26.

Bosch, R.F. and White, W.B. 2004. Lithofacies andTransport of Clastic Sediments in Karstic Aquifers. In: Sasowsky, I.D. and Mylroie, J. (eds.), Studies of Cave Sediments, 1-22.

Cañaveras, J.C., Sánchez-Moral, S., Lario, J., Cuezva, S., Fernández, A. and Muñoz, M.C. 2011. El modelo de relleno, o cómo llegaron los restos a la Galería del Osario. In: Rasilla, M., Rosas, A., Cañaveras, J.C. and Lalueza-Fox, C. (eds.), La cueva de El Sidrón (Borines, Piloña, Asturias). Investigación interdisciplinar de un grupo neandertal. Excavaciones Arqueológicas en Asturias, Monografías I, Consejería de Cultura y Turismo. Oviedo, 43-63.

Dunhan, R.J. 1962. Classification of Carbonate rocks according to depositional texture. In: Ham, W.E. (ed.) Classification of carbonate rocks. AAPG Mem. 1, 108-121.

Farrand,W.R. 2001. Sediments and stratigraphy in rockshelters and caves: A personal perspective on principles and pragmatic. In: Woodward, J. C. and Goldberg, P. (eds.), Rockshelter sediment Records and Environmental Change in the Mediterranean Region, Geoarchaeology, 16, Special Issue, 537-557.

Folk, R.L. 1962. Spectral subdivision of limestones types. In: Ham, W.E. (ed.) Classification of carbonate rocks. AAPG Mem. 1, 62-84.

Ford, D. and Williams, P.D. 2007. Karst Hydrogeology and Geomorphology. Wiley, 576 pp.

Fortea, J., Rasilla de la, M., Martínez, E., Sánchez-Moral, S., Cañaveras, J.C., Cuezva, S., Rosas, A., Soler, V., Julià, R., Torres De, T., Ortiz, J.E., Castro, J., Badal, E., Altuna, J. y Alonso, J. 2003. La cueva de El Sidrón (Borines, Piloña, Asturias): Primeros resultados. Estudios Geológicos, 59, 159-179.

Fortea, J., Rasilla de la, M., Martínez, E., Sánchez-Moral, S., Cañaveras, J.C., Cuezva, S., Rosas, A., Soler, V., Julià, R., Torres de, T., Ortiz, J.E., Castro, J., Badal, E., Altuna, J. and Alonso, J. 2007a. La cueva de El Sidrón (Borines, Piloña, Asturias): Campañas arqueológicas de 2000 a 2002. Excavaciones Arqueológicas en Asturias 19992002, 5, 191-205.

Fortea, J., Rasilla, M. De la, d. Santamaría, D., Martínez, L., Duarte, E., Fernández de la Vega, J., Martínez, E., Cañaveras, J.C., Sánchez-Moral, S., Cuezva, S., Lario, J., Rosas, A., Martínez-Maza, C., García-Tabernero, A.,
Bastir, M., Huguet, R., Estalrrich, A., García-Vargas, S., Sánchez-Meseguer, A., León, S., Lalueza-Fox, C., Torres, T. De, Ortiz, J. E., Julià, R., Grün, R., Valladas, H., Mercier, N., Tisnèrat-Laborde, N., Soler, V., Silva, P.G., Carrasco, P., Ayarza, P., Álvarez, F., Santos-Delgado, G., Altuna, J., Badal, E. and Alonso, J. 2007b. La cueva de El Sidrón (Borines, Piloña, Asturias). Campañas arqueológicas de 2003-2006. Excavaciones arqueológicas en Asturias 2003 a 2006, 6, 367-384.

Fortea, J., Rasilla, M. de la, Santamaría, D., Rosas, A., Lalueza-Fox, C., Martínez, E., Sánchez-Moral, S. y Cañaveras, J. C. 2007c. El Sidrón (Borines, Piloña, Asturias). La presencia del Homo neanderthalensis en Asturias. La Prehistoria en Asturias. Un legado artístico único en el mundo. La Nueva España, Oviedo, 321-354.

Goldberg, P. and Macphail, R.I. 2005. Practical and theoretical geoarchaeology. Wiley-Blackwell, 468 pp.

Goldberg, P. and Sherwood, S.D. 2006. Deciphering human prehistory through the geoarcheological study of cave sediments. Evolutionary Anthropology, 15, 20-36.

Jordá Pardo, J.F. 2009. Geoarqueología, de la teoría a la práctica: interpretación de un yacimiento de la Edad del Hierro en la Península Ibérica. Xama, 19-23, 45-61.

Martínez García, E. 2011. Evolución geológica de la zona de EI Sidrón y sus áreas limítrofes. In: Rasilla, M., Rosas, A., Cañaveras, J.C. and Lalueza-Fox, C. (eds.), La cueva de El Sidrón (Borines, Piloña, Asturias). Investigación interdisciplinar de un grupo neandertal. Excavaciones Arqueológicas en Asturias, I, 29-33.

Pettijohn, F.J., Potter, P.E. and Siever, R. 1973. Sand and Sandstone. Springer-Verlag, New York. 312 pp.

Prieto, J. L., Abenza, J.M., Montes, R., Sanguino, J. and Muñoz, E. 2001. Hallazgos antropológicos y arqueológicos en el complejo kárstico de El Sidrón (Vallobal, Infiesto, Concejo de Piloña, Asturias). Munibe, 53, 19-29.

Rasilla, M. de la, Santamaría, D. Torres, T. Ortiz, J. E., Valladas, H. Mercier, N., Tisnérat-Laborde, N., Grün, R., Eggins, S., Juliá, R., Soler, V., Lalueza, C., Rosas, A., Silva, P.G., Sánchez-Moral, S. and Cañaveras J.C. 2013. La cronometría de la cueva de El Sidrón (Piloña, Asturias), 306-308.

Rasilla, M. de la, Rosas, A., Cañaveras, J.C. and Lalueza-Fox, C. (eds.) 2011. La cueva de El Sidrón (Borines, Piloña, Asturias). Investigación interdisciplinar de un grupo neandertal. Consejería de Cultura y Turismo, Gobierno del Principado de Asturias y TRABE SLU, Oviedo, 211 pp.

Rasilla, M. de la, Rosas, A., Cañaveras, J. C., Lalueza, C., Santamaría, D., Sánchez Moral, S., Estalrrich, A., García Tabernero, A., Silva, P. G., Martínez, E., Santos-Delgado, G., Martínez, L., Duarte, E., Huguet, R., Bastir, M., Fernández de la Vega, F., Suárez, P., Díez, A. B., Fernández Cascón, B., Cuezva, S., Fernández Cortés, A., García Antón, E., Muñoz, C., Lario, J., Carrasco, P., Huerta, P., Ayarza, P., Álvarez Lobato, F., Rodríguez, L., Picón, I., Fernández, B., Standing, M., Sesé, C., Torres, T. de, Ortiz, J. E., Valladas, H., Mercier, N., Tisnèrat-Laborde, N., Grün, R., Eggins, S., Higham, T., Wood, R. E., Julià, R., Soler, V., Badal, E., Tarriño, A., Salazar, D. C. and Alonso, J. 2014. La cueva de El Sidrón (Piloña, Asturias). In: Sala, R. (ed.), Carbonell, E., Bermúdez de Castro, J. M. and 
Arsuaga, J. L. (coords.). Los cazadores recolectores del Pleistoceno y del Holoceno en Iberia y el Estrecho de Gibraltar: Estado actual del conocimiento del registro arqueológico. Universidad de Burgos y Fundación Atapuerca. Burgos, 122-128.

Rosas, A. and Aguirre, E. 1999. Restos humanos neandertales de la cueva del Sidrón, Piloña, Asturias. Nota preliminar. Estudios Geológicos 55, 181-190.

Rosas, A., Martínez Maza, C., Bastir, M., García-Tabernero, A., Lalueza-Fox, C., Huguet, R., Ortiz, J. E., Julià, R., Soler, V., Torres, T. de, Cuezva, S., Martínez, E., Lario, J., Cañaveras, J. C., Sánchez-Moral, S., Santamaría, D., Rasilla, M. de la and Fortea, J. 2006. Paleobiology and comparative morphology of a late Neandertal sample from El Sidrón (Asturias, Spain). Proceedings National Academy Sciences USA, 103, 19266-19271.

Rosas A., Martínez-Maza C., Bastir M., García-Tabernero A., Lalueza-Fox C., Huguet R., Estalrrich A., García-Vargas S., de la Rasilla M. and Fortea J. 2007: Paleobiological aspects of El Sidrón (Asturias, Spain) Neandertals. American Journal of Physical Anthropology, S44, 202.

Rosas, A.; Estalrrich, A.; García-Vargas, S.; GarcíaTabernero, A.; Huguet, R.; Lalueza-Fox, C., Rasilla, M. de la and Fortea, J. 2013. Identification of Neandertal individuals in fragmentary fossil assemblages by means of teeth associations. The case of the El Sidrón (Asturias, Spain). Comptes Rendus Palevol, 12 (5), 279-291.

Sánchez-Moral, S.; Cañaveras, J. C.; Lario, J.; Cuezva, S.; Silva, P. G.; Rasilla, M. de la and Fortea, J. 2007. Caracterización del relleno sedimentario de la Galería del Osario (cueva de El Sidrón, Asturias, España). In: Lario, J. y Silva P.G. (eds.), XII Reunión Nacional de Cuaternario, AEQUA, 123-124.

Santamaría, D. 2012. La transición del Paleolítico Medio al Superior en Asturias. El abrigo de La Viña (La Manzaneda, Oviedo) y la cueva de El Sidrón (Borines, Piloña). Servicio de Publicaciones de la Universidad de Oviedo. Oviedo. 1518 pp.

Santamaría, D. Fortea, J. Rasilla, M. de la, Martínez, L., Martínez, E., Cañaveras, J. C., Sánchez-Moral, S., Rosas, A, Estalrrich, A, García-Tabernero, A. and Lalueza-Fox, C. 2010. The technological and typological behaviour of a Neanderthal group from El Sidrón Cave (Asturias, Spain). Oxford Journal of Archaeology, 29 (2), 119-148.

Santamaría, D., Martínez, L., Duarte, E., Cañaveras, J. C., Rosas, A., Sánchez-Moral, S., Silva, P. G., Lalueza-Fox, C. and Rasilla, M. de la. 2011. Los remontajes líticos muste- rienses de la cueva de El Sidrón (Borines, Piloña, Asturias). In: Turu, V. and Constante, A. (eds.). EI Cuaternario en España y áreas afines, avances en 2011. XIII Reunión Nacional de Cuaternario (Andorra, 2011). AEQUA y Fundación Marcel Chevalier. Andorra la Vella, 229-233.

Santos-Delgado, G., Martínez, J., Silva, P. G., SánchezMoral, S., Cañaveras, J. C. and Rasilla, M. de la. 2012a. Contribución al conocimiento de la cueva de El Sidrón (Piloña, Asturias) con técnicas de láser escáner 3D. In: González-Díez, A. (coord.), Avances de la Geomorfología en España 2010-2012. PubliCan-Ediciones de la Universidad de Cantabria: 255-258.

Santos-Delgado, G., Silva, P. G., Standing, M., Huerta, P., Fernández, B., Cañaveras, J. C., Sánchez-Moral, S. and Rasilla, M. de la. 2012b. Análisis topográfico y geomorfológico del complejo kárstico de El Sidrón (Piloña, Asturias). Geo-Temas, 13: 224.

Silva, P. G., Santos, G., Carrasco, P., Huerta, P., Ayarza, P., Álvarez Lobato, F., Fernández Macarro, B. and Standing, M. 2011. La geomorfología, topografía y prospección geofísica del complejo de El Sidrón. La búsqueda del lugar de procedencia de los restos fósiles. In: Rasilla, M., Rosas, A., Cañaveras, J.C. y Lalueza-Fox, C. (eds.), La cueva de El Sidrón (Borines, Piloña, Asturias). Investigación interdisciplinar de un grupo neandertal. Consejería de Cultura y Turismo. Oviedo, 65-79.

Torres, T., Ortiz, J. E., Grün, R., Eggins, S., Valladas, H., Mercier, N., Tisnérat-Laborde, N., Juliá, R., Soler, V., Martínez, E., Sánchez-Moral, S., Cañaveras, J. C., Lario, J., Badal, E., Lalueza-Fox, C., Rosas, A., Santamaría, D., de la Rasilla, M. and Fortea, J. 2010. Dating of the hominid (Homo neanderthalensis) remains accumulation from El Sidrón Cave (Piloña, Asturias, North Spain): an example of a multimethodological approach to the dating of Upper Pleistocene sites. Archaeometry, 52, 680-705.

Wood, R. E., Higham, T. F. G., Torres, T de, Tisnérat-Laborde, N., Valladas, H., Ortiz, J.E., Lalueza, C., Sánchez-Moral, S., Cañaveras, J.C., Rosas, A., Santamaría, D. and Rasilla, M. de la. 2013. A new date for the Neanderthals from El Sidrón Cave (Asturias, Northern Spain). Archaeometry, 55, 148-158.

Woodward, J. C. and Goldberg, P. 2001.The sedimentary records in Mediterranean rockshelters and caves: archives of environmental change. Geoarchaeology, 16 (4), 327-354.

Recibido: marzo 2015

Revisado: junio 2015

Aceptado: diciembre 2015

Publicado: marzo 2018 